\title{
Point-to-Hyperplane Nearest Neighbor Search Beyond the Unit Hypersphere
}

\author{
Qiang Huang \\ School of Computing \\ National University of Singapore \\ huangq@comp.nus.edu.sg
}

\author{
Yifan Lei \\ School of Computing \\ National University of Singapore \\ leiyifan@comp.nus.edu.sg
}

\author{
Anthony K. H. Tung \\ School of Computing \\ National University of Singapore \\ atung@comp.nus.edu.sg
}

\begin{abstract}
Point-to-Hyperplane Nearest Neighbor Search (P2HNNS) is a fundamental yet challenging problem, and it has plenty of applications in various fields. Existing hyperplane hashing schemes enjoy sublinear query time and achieve excellent performance on applications such as large-scale active learning with Support Vector Machines (SVMs). However, they only conditionally deal with this problem with a strong assumption that all of the data objects are normalized, located at the unit hypersphere. Those hyperplane hashing schemes may be arbitrarily bad without this assumption.

In this paper, we introduce a new asymmetric transformation and develop the first two provable hyperplane hashing schemes, Nearest Hyperplane hashing (NH) and Furthest Hyperplane hashing (FH), for high-dimensional P2HNNS beyond the unit hypersphere. With this asymmetric transformation, we demonstrate that the hash functions of $\mathrm{NH}$ and $\mathrm{FH}$ are locality-sensitive to the hyperplane queries, and both of them enjoy quality guarantee on query results. Moreover, we propose a data-dependent multi-partition strategy to boost the search performance of $\mathrm{FH}$. NH can perform the hyperplane queries in sub-linear time, while $\mathrm{FH}$ enjoys a better practical performance. We evaluate $\mathrm{NH}$ and $\mathrm{FH}$ over five real-life datasets and show that we are around $3 \sim 100 \times$ faster than the best competitor in four out of five datasets, especially for the recall in $[20 \%, 80 \%]$. Code is available at https://github.com/HuangQiang/P2HNNS.
\end{abstract}

\section{CCS CONCEPTS}

- Information systems $\rightarrow$ Nearest-neighbor search; • Theory of computation $\rightarrow$ Nearest neighbor algorithms.

\section{KEYWORDS}

Locality-Sensitive Hashing; Nearest Neighbor Search; Furthest Neighbor Search; Asymmetric Transformation; Active Learning

\section{ACM Reference Format:}

Qiang Huang, Yifan Lei, and Anthony K. H. Tung. 2021. Point-to-Hyperplane Nearest Neighbor Search Beyond the Unit Hypersphere. In Proceedings of the 2021 International Conference on Management of Data (SIGMOD '21), fune 20-25, 2021, Virtual Event, China. ACM, New York, NY, USA, 13 pages. https://doi.org/10.1145/3448016.3457240

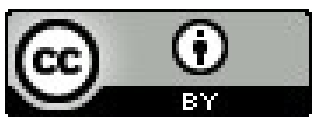

This work is licensed under a Creative Commons Attribution International 4.0 License SIGMOD '21, Fune 20-25, 2021, Virtual Event, China. (C) 2021 Copyright held by the owner/author(s). ACM ISBN 978-1-4503-8343-1/21/06.

https://doi.org/10.1145/3448016.3457240

\section{INTRODUCTION}

Nearest Neighbor Search (NNS) over high-dimensional spaces is of vital importance in many areas, such as databases, data mining, and artificial intelligence. Locality-Sensitive Hashing (LSH) [13, 19] and its variants $[1,3,8,11]$ have been one of the most popular methods for high-dimensional NNS due to their excellent practical performance and robust theoretical guarantees on sub-linear query time and query results. However, most of the existing methods $[2,12,15,16,21-23,26,27,37,38,43,49,50]$ mainly deal with the classic point-to-point NNS (or simply NNS), that is, both queries and data objects are points in the same dimension.

In this paper, we study a fundamental yet more difficult problem, named Point-to-Hyperplane NNS (P2HNNS), which has plenty of applications in large-scale active learning with SVMs [34, 39, 41], maximum margin clustering [47, 48], and large-margin dimensionality reduction [33, 45]. Given a database $\mathcal{D}$ of $n$ data objects represented as points in a $(d-1)$-dimensional Euclidean space $\mathbb{R}^{d-1}$ and a hyperplane query $\boldsymbol{q}=\left(q_{1}, \cdots, q_{d}\right) \in \mathbb{R}^{d}$ such that $q_{d}+\sum_{i=1}^{d-1} x_{i} q_{i}=0$ for any point $x=\left(x_{1}, \cdots, x_{d-1}\right) \in \mathbb{R}^{d-1}$ at this hyperplane, P2HNNS aims to find the nearest data point $\boldsymbol{p}^{*} \in \mathcal{D}$ of $\boldsymbol{q}$ such that

$$
\boldsymbol{p}^{*}=\arg \min _{\boldsymbol{p} \in \mathcal{D}} d_{P 2 H}(\boldsymbol{p}, \boldsymbol{q}),
$$

where $d_{P 2 H}(\boldsymbol{p}, \boldsymbol{q})$ represents the distance from a data point $\boldsymbol{p}$ to the hyperplane query $\boldsymbol{q}$. For any $\boldsymbol{p}=\left(p_{1}, \cdots, p_{d-1}\right)$ and $\boldsymbol{q}=$ $\left(q_{1}, \cdots, q_{d}\right), d_{P 2 H}(\boldsymbol{p}, \boldsymbol{q})$ is computed as follows:

$$
d_{P 2 H}(\boldsymbol{p}, \boldsymbol{q})=\frac{\left|q_{d}+\sum_{i=1}^{d-1} p_{i} q_{i}\right|}{\sqrt{\sum_{i=1}^{d-1} q_{i}^{2}}} .
$$

There are many hyperplane hashing schemes [4, 20, 24, 25, 42] to deal with the problem of high-dimensional P2HNNS. Jain et al. $[20,42]$ introduced the first two provable hashing schemes Angle Hyperplane hashing $(\mathrm{AH})$ and Embedding Hyperplane hashing (EH) with two families of linear hash functions which are localitysensitive to the angular distance between data objects and the normal vector of the hyperplane query. Later, researchers proposed the Bilinear Hyperplane hashing (BH) [24] and Multilinear Hyperplane hashing (MH) [25], which amplifies the collision probabilities between different angular distances using the bilinear hash functions and multilinear hash functions, respectively. By exploiting the asymmetry hash functions between data objects and hyperplane queries, Aumüller et al. developed a general distance-sensitive hashing [4] beyond LSH. All of these methods use the static $(K, L)$-bucketing strategy [19] for high-dimensional P2HNNS, where $K$ is the number of concatenated hash functions; $L$ is the number of hash tables.

However, these hyperplane hashing schemes [4, 20, 24, 25, 42] only conditionally deal with this problem with a strong assumption: 
all of the data are located at the unit hypersphere, i.e., the Euclidean norm $\|\boldsymbol{p}\|=\sqrt{\sum_{i=1}^{d-1} p_{i}^{2}}=1$ for all $\boldsymbol{p} \in \mathcal{D} \subset \mathbb{R}^{d-1}$. They work well in large-scale active learning [20, 24, 25]. However, in most applications, such as clustering $[47,48]$ and dimension reduction $[33,45]$, data objects are often not normalized. For such applications, these hyperplane hashing schemes may be arbitrarily bad. Let $\boldsymbol{w}_{\boldsymbol{q}}=$ $\left(q_{1}, \cdots, q_{d-1}\right)$ be the normal vector of the hyperplane query $\boldsymbol{q}$. The reason is that, if this assumption fails, the locality-sensitive property to the angular distance $\alpha_{\boldsymbol{p}, \boldsymbol{w}_{\boldsymbol{q}}}$ between $\boldsymbol{p}$ and $\boldsymbol{w}_{\boldsymbol{q}}$ will be no longer valid, and hence the retrieved identities will not follow the order of $\arg \min _{\boldsymbol{p} \in \mathcal{D}} d_{P 2 H}(\boldsymbol{p}, \boldsymbol{q})$, i.e.,

$$
\arg \min _{\boldsymbol{p} \in \mathcal{D}} \frac{\left|\sum_{i=1}^{d-1} p_{i} q_{i}\right|}{\|\boldsymbol{p}\|\left\|\boldsymbol{w}_{\boldsymbol{q}}\right\|} \Leftrightarrow \arg \min _{\boldsymbol{p} \in \mathcal{D}} d_{P 2 H}(\boldsymbol{p}, \boldsymbol{q}) .
$$

To the best of our knowledge, there is no existing hyperplane hashing scheme which can deal with the P2HNNS in sub-linear time without the unit hypersphere assumption of the data objects.

Moreover, $\rho$ is a key performance indicator for the static $(K, L)$ bucketing setting, where $\rho=\ln \left(1 / p_{1}\right) / \ln \left(1 / p_{2}\right) ; p_{1}$ and $p_{2}$ are the positive and negative collision probabilities, respectively. The hyperplane hashing schemes [4, 20, 24, 25, 42] with this setting require $L=O\left(n^{\rho}\right)$ hash tables to achieve a constant success probability for P2HNNS. The $\rho$ value can be small when $d_{P 2 H}(\boldsymbol{p}, \boldsymbol{q})$ is large. However, as summarized in [25], the $p_{1}$ and $p_{2}$ values of $\mathrm{AH}, \mathrm{EH}, \mathrm{BH}$, and $\mathrm{MH}$ are less than $\frac{1}{2}$, i.e., $0<p_{2}<p_{1}<\frac{1}{2}$. When $d_{P 2 H}(\boldsymbol{p}, \boldsymbol{q}) \rightarrow 0, \lim _{d_{P 2 H} \rightarrow 0} p_{1}=\lim _{d_{P 2 H} \rightarrow 0} p_{2}=\frac{1}{2}$, then $\rho=\lim _{d_{P 2 H} \rightarrow 0} \ln \left(1 / p_{1}\right) / \ln \left(1 / p_{2}\right)=1$, leading to $L=O(n)$ hash tables. Therefore, even though they enjoy sub-linear query time, their practical space and query time overhead can be very large.

\subsection{Our Contributions}

To deal with the high-dimensional P2HNNS beyond the unit hypersphere, we propose a novel asymmetric transformation which converts this challenging problem into the classic NNS problem. With a state-of-the-art LSH scheme based on Longest Circular Co-Substring (LCCS-LSH) [22], we develop the first provably sublinear time method Nearest Hyperplane hashing $(\mathrm{NH})$ for P2HNNS without the data normalization assumption. We show the hash functions of $\mathrm{NH}$ enjoy the locality-sensitive property to the hyperplane queries: the collision probability between any $\boldsymbol{p}$ and $\boldsymbol{q}$ increases as $d_{P 2 H}(\boldsymbol{p}, \boldsymbol{q})$ decreases. NH can deal with the P2HNNS in sub-quadratic space and sub-linear query time.

With a minor modification of this asymmetric transformation, the P2HNNS problem can be transformed to another classic problem of point-to-point Furthest Neighbor Search (or simply FNS). By adopting a state-of-the-art LSH scheme Reverse Query-Aware LSH (RQALSH) [14] for FNS, we develop another provable method Furthest Hyperplane hashing (FH) for P2HNNS. The hash functions of $\mathrm{FH}$ also have the locality-sensitive property such that the probability with which any $\boldsymbol{p}$ and $\boldsymbol{q}$ are separated into different buckets increases as $d_{P 2 H}(\boldsymbol{p}, \boldsymbol{q})$ decreases. Moreover, we propose a datadependent multi-partition strategy to boost the search performance of $\mathrm{FH}$, which enjoys better practical performance than $\mathrm{NH}$.

We provide a comprehensive comparison of $\mathrm{NH}$ and $\mathrm{FH}$ with four different methodologies, which includes two state-of-the-art methods $\mathrm{BH}$ and $\mathrm{MH}$ and two trivial baselines Random-Scan and
Sorted-Scan. The results over five real-life datasets show that we are around $3 \sim 100 \times$ faster than the best competitor in four out of five datasets, especially when the recall falls in the range of $[20 \%, 80 \%]$.

\subsection{Related Work}

P2HNNS. A trivial solution for P2HNNS is the exhaustive linear scan, but its query time $O(n d)$ is inefficient when $n$ or $d$ is large. For the data in low to medium dimensions (e.g., $d \leq 10$ ), this problem can be well-solved by adapting vanilla tree structures (e.g., BallTree [29], Cone-Tree [32], and KD-Tree [6]) with the branch-andbound space partitioning strategy. However, due to the "curse of dimensionality," the space and/or query time complexities of these methods are highly dependent on $d$ and their expansion constants, which are not suitable in high-dimensional spaces [7, 44].

Hyperplane hashing schemes [4, 20, 24, 25, 42] have been proposed recently for high-dimensional P2HNNS. In [20, 42], Jain et al. designed two families of linear hash functions which are locality-

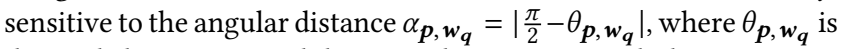
the angle between $\boldsymbol{p}$ and the normal vector $\boldsymbol{w}_{\boldsymbol{q}}$. With these two LSH families, they proposed the first two hyperplane hashing schemes $\mathrm{AH}$ and $\mathrm{EH}$ for P2HNNS with theoretical guarantees. To enhance the locality-sensitive property with a larger difference of the collision probabilities bwtween different $\alpha_{\boldsymbol{o}, \boldsymbol{w}_{\boldsymbol{q}}}$ values, BH [24] and $\mathrm{MH}$ [25] have been developed with the newly designed bilinear hash functions and multilinear hash functions. Moreover, the researchers developed two learning based methods to boost the search performance of $\mathrm{BH}$ and $\mathrm{MH}$, respectively.

Recently, Aumüller et al. proposed a new distance-sensitive hashing scheme [4] beyond LSH for P2HNNS, with an asymmetry design of a pair of hash functions for data objects and hyperplane queries. Compared with the linear scan, these schemes enjoy comparable accuracy of retrieved results and sub-linear query time by reducing the number of data objects to check in orders of magnitude.

Asymmetric Transformation. For the problem of Maximum Inner Product Search (MIPS), there exists many asymmetric LSH schemes which design different asymmetric transformations to address the problem of data normalization. L2-ALSH [35] and SignALSH [36] use the asymmetric transformations by extending data objects and queries with several dimensions, but they contain additive error. XBOX [5] develops an exact asymmetric transformation with only one dimension extension. Simple-LSH [28] adopts a transformation which maps all data objects and queries to the same hypersphere to reduce the distortion error. Recently, H2-ALSH [17] and Norm-Ranging LSH [46] integrate the data partition into the asymmetric transformations to further improve the MIPS performance. The key of these schemes is to design asymmetric transformations for data objects and queries, so that the (asymmetric) hash functions could be locality-sensitive to the inner product.

According to Equation 7 which will be illustrated later, the P2HNNS can be regarded as a minimum absolute inner product search problem. Both problems are related to the inner product, but the P2HNNS contains an extra absolute value operator. Thus, existing asymmetric transformations for MIPS cannot be used directly for P2HNNS, but they motivate us to develop new transformations for data points and hyperplane queries to remove the absolute value operator and address the data normalization problem. 


\section{PRELIMINARIES}

In this work, we convert the P2HNNS into the classic point-to-point NNS (or FNS) problem. For those two problems, both data objects and queries are represented as points in the same dimension.

\subsection{Locality-Sensitive Hashing}

LSH schemes are one of the most popular methods for high dimensional NNS $[2,11,12,22]$. Given a hash function $h$, we say two objects $\boldsymbol{o}$ and $\boldsymbol{q}$ collide in the same bucket if $h(\boldsymbol{o})=h(\boldsymbol{q})$. Let $\operatorname{Dist}(\boldsymbol{o}, \boldsymbol{q})$ be a distance function between any two $\boldsymbol{o}$ and $\boldsymbol{q}$. We first define a modified LSH family in a form which will be used later.

Definition 2.1 ((Asymmetric) LSH Family): Given a search radius $R(R>0)$ and an approximation ratio $c$, a family of hash functions $\mathcal{H}$, along with two vector transformations $\boldsymbol{P}: \mathbb{R}^{d} \rightarrow \mathbb{R}^{d^{\prime}}$ and $Q:$ $\mathbb{R}^{d} \rightarrow \mathbb{R}^{d^{\prime}}$, is called $\left(R, c R, p_{1}, p_{2}\right)$-sensitive for Dist $(\cdot, \cdot)$ if, for any $\boldsymbol{o}, \boldsymbol{q} \in \mathbb{R}^{d}$, the hash function $h$ drawn uniformly from $\mathcal{H}$ satisfies the following conditions:

- $\operatorname{Dist}(\boldsymbol{o}, \boldsymbol{q}) \leq R \Rightarrow \operatorname{Pr}[h(\boldsymbol{P}(\boldsymbol{o}))=h(\boldsymbol{Q}(\boldsymbol{q}))] \geq p_{1}$;

- $\operatorname{Dist}(\boldsymbol{o}, \boldsymbol{q}) \geq c R \Rightarrow \operatorname{Pr}[h(\boldsymbol{P}(\boldsymbol{o}))=h(\boldsymbol{Q}(\boldsymbol{q}))] \leq p_{2}$;

- $p_{1}>p_{2}$ and $c>1$.

Note that $P$ is only applied to the data objects, while $Q$ is only applied to the hyperplane queries; when $Q(x)=P(x)=x$, Definition 2.1 degenerates to the vanilla definition of LSH family.

LCCS-LSH. LCCS-LSH [22] is a state-of-the-art LSH scheme for NNS. To deal with the Euclidean distance, LCCS-LSH uses the popular random projection LSH family [11], whose LSH function is defined as follows:

$$
h_{\boldsymbol{a}, b}(\boldsymbol{o})=\left\lfloor\frac{\langle\boldsymbol{a}, \boldsymbol{o}\rangle+b}{w}\right\rfloor,
$$

where $\boldsymbol{a}$ is a random vector with each entry drawn i.i.d. from normal distribution, i.e., $a_{i} \sim \mathcal{N}(0,1) ; w$ is a pre-specified bucket width; $b$ is a random offset chosen uniformly at random from $[0, w)$.

Let $s=\|\boldsymbol{o}-\boldsymbol{q}\|=\sqrt{\sum_{i=1}^{d}\left(o_{i}-q_{i}\right)^{2}}$ be the Euclidean distance between any two $\boldsymbol{o}=\left(o_{1}, \cdots, o_{d}\right)$ and $\boldsymbol{q}=\left(q_{1}, \cdots, q_{d}\right)$. The collision probability $p(s)$ is computed as follows [11]:

$$
\begin{aligned}
p(s) & =\operatorname{Pr}\left[h_{\boldsymbol{a}, b}(\boldsymbol{o})=h_{\boldsymbol{a}, b}(\boldsymbol{q})\right] \\
& =1-2 \Phi(-w / s)-\frac{2}{\sqrt{2 \pi}(w / s)}\left(1-e^{-(w / s)^{2} / 2}\right),
\end{aligned}
$$

where $\Phi(x)=\int_{-\infty}^{x} \frac{1}{\sqrt{2 \pi}} e^{-x^{2} / 2} d x$ is the Cumulative Distribution Function (CDF) of $\mathcal{N}(0,1)$.

Let $p_{1}=p(1)$ and $p_{2}=p(c)$, where $p(\cdot)$ is defined by Equation 4. We have Theorem 2.1 for LCCS-LSH for NNS as follows [22]:

Theorem 2.1: Setting $t=O\left(n^{\rho}\right)$. Given an approximation ratio $c$ (c $>1$ ), LCCS-LSH is a data structure which can find an $\boldsymbol{o} \in \mathcal{D}$ such that $\|\boldsymbol{o}-\boldsymbol{q}\| \leq c \cdot\left\|\boldsymbol{o}^{*}-\boldsymbol{q}\right\|$ with probability at least $1 / 4$ in $O\left(n^{1+\rho}\right)$ space, $O\left(n^{1+\rho}(d+\log n)\right)$ indexing time, and $O\left(n^{\rho}(d+\log n)\right)$ query time, where $\rho=\ln \left(1 / p_{1}\right) / \ln \left(1 / p_{2}\right)$ and $\boldsymbol{o}^{*}=\arg \min _{\boldsymbol{o} \in \mathcal{D}}\|\boldsymbol{o}-\boldsymbol{q}\|$.

\subsection{Reverse Locality-Sensitive Hashing}

As the LSH family designed for NNS, there exists a concept of Reverse LSH (RLSH) family [14] designed for FNS, which is a set of hash functions with the locality-sensitive property that dissimilar objects have a higher probability of being separated into different buckets than the similar ones. Given a hash function $h, \boldsymbol{o}$ and $\boldsymbol{q}$ are separated into different buckets if $h(\boldsymbol{o}) \neq h(\boldsymbol{q})$. Next, we define a modified RLSH family in a form which will be used later.

Definition 2.2 ((Asymmetric) RLSH Family): Given a search radius $R(R>0)$ and an approximation ratio $c$, a family of hash functions $\mathcal{H}$, along with two vector transformations $\boldsymbol{P}: \mathbb{R}^{d} \rightarrow \mathbb{R}^{d^{\prime}}$ and $Q$ : $\mathbb{R}^{d} \rightarrow \mathbb{R}^{d^{\prime}}$, is called $\left(R, \frac{R}{c}, \xi_{1}, \xi_{2}\right)$-sensitive for $\operatorname{Dist}(\cdot, \cdot)$ if, for any $\boldsymbol{o}, \boldsymbol{q} \in \mathbb{R}^{d}$, the hash function $h$ drawn uniformly from $\mathcal{H}$ satisfies the following conditions:

- $\operatorname{Dist}(\boldsymbol{o}, \boldsymbol{q}) \geq R \Rightarrow \operatorname{Pr}[h(\boldsymbol{P}(\boldsymbol{o})) \neq h(\boldsymbol{Q}(\boldsymbol{q}))] \geq \xi_{1}$;

- $\operatorname{Dist}(\boldsymbol{o}, \boldsymbol{q}) \leq \frac{R}{c} \Rightarrow \operatorname{Pr}[h(\boldsymbol{P}(\boldsymbol{o})) \neq h(\boldsymbol{Q}(\boldsymbol{q}))] \leq \xi_{2}$;

- $\xi_{1}>\xi_{2}$ and $c>1$.

Similar to Definition 2.1, $\boldsymbol{P}$ is only applied to data objects, and $Q$ is only applied to hyperplane queries; when $Q(x)=P(x)=x$, Definition 2.2 degenerates to the vanilla definition of RLSH family.

RQALSH. RQALSH [14] is one of the most efficient hashing schemes for high-dimensional FNS [9, 10, 14, 18, 30,31]. Its family is a typical RLSH family designed for Euclidean distance. Let $\boldsymbol{a}$ be a random vector with each entry drawn i.i.d. from normal distribution, i.e., $a_{i} \sim \mathcal{N}(0,1)$. The RQALSH function is defined as follows [14]:

$$
h_{\boldsymbol{a}}(\boldsymbol{o})=\langle\boldsymbol{a}, \boldsymbol{o}\rangle \text {. }
$$

Let $w$ be a pre-specified bucket width. We say $\boldsymbol{o}$ and $\boldsymbol{q}$ are separated into different buckets under $h_{\boldsymbol{a}}$ if $\left|h_{\boldsymbol{a}}(\boldsymbol{o})-h_{\boldsymbol{a}}(\boldsymbol{q})\right|>\frac{w}{2}$. Let $s=\|\boldsymbol{o}-\boldsymbol{q}\|$ be the Euclidean distance between any two $\boldsymbol{o}, \boldsymbol{q} \in \mathbb{R}^{d}$. The separation probability $\xi(s)$ is computed as follows [14]:

$$
\xi(s)=\operatorname{Pr}\left[\left|h_{\boldsymbol{a}}(\boldsymbol{o})-h_{\boldsymbol{a}}(\boldsymbol{q})\right|>\frac{w}{2}\right]=2 \Phi\left(-\frac{w}{2 s}\right) .
$$

Let $\xi_{1}=\xi(1)$ and $\xi_{2}=\xi\left(\frac{1}{c}\right)$, where $\xi(\cdot)$ is defined by Equation 6. We have Theorem 2.2 for RQALSH for FNS as follows [14]:

Theorem 2.2: Let $m=O(\log n)$ and $l=O(\log n)(l \leq m)$. Given an approximation ratio $c(c>1), R Q A L S H$ is a data structure which can find an $\boldsymbol{o} \in \mathcal{D}$ such that $\|\boldsymbol{o}-\boldsymbol{q}\| \geq\left\|\boldsymbol{o}^{*}-\boldsymbol{q}\right\| / \mathrm{c}$ with constant probability in $O(n \log n)$ space, $O((d+\log n) n \log n)$ indexing time, and $O((d+n) \log n)$ query time, where $\boldsymbol{o}^{*}=\arg \max _{\boldsymbol{o} \in \mathcal{D}}\|\boldsymbol{o}-\boldsymbol{q}\|$.

\section{HYPERPLANE HASHING SCHEMES}

\subsection{Overview}

The problem of P2HNNS is difficult because the data is often not normalized and there exists an absolute value operator in Equation 2. Thus, we develop some transformations on data objects and the hyperplane queries to make this problem easier to deal with.

First of all, we extend the data objects with one dimension so that both data and queries have the same dimension. Then, we show there is no LSH and RLSH families for $d_{P 2 H}(\cdot, \cdot)$. Thus, we design an asymmetric transformation to convert the P2HNNS into the classic NNS (or FNS). The asymmetric transformation consists of two steps: object embedding and hypersphere transformation. The step of object embedding can remove the absolute value operator, while the step of hypersphere transformation can address the problem of data normalization. With different designs of the hypersphere transformation, we propose two schemes Nearest Hyperplane hashing $(\mathrm{NH})$ and Furthest Hyperplane hashing $(\mathrm{FH})$ for P2HNNS. An overview of NH and FH is depicted in Figure 1. 

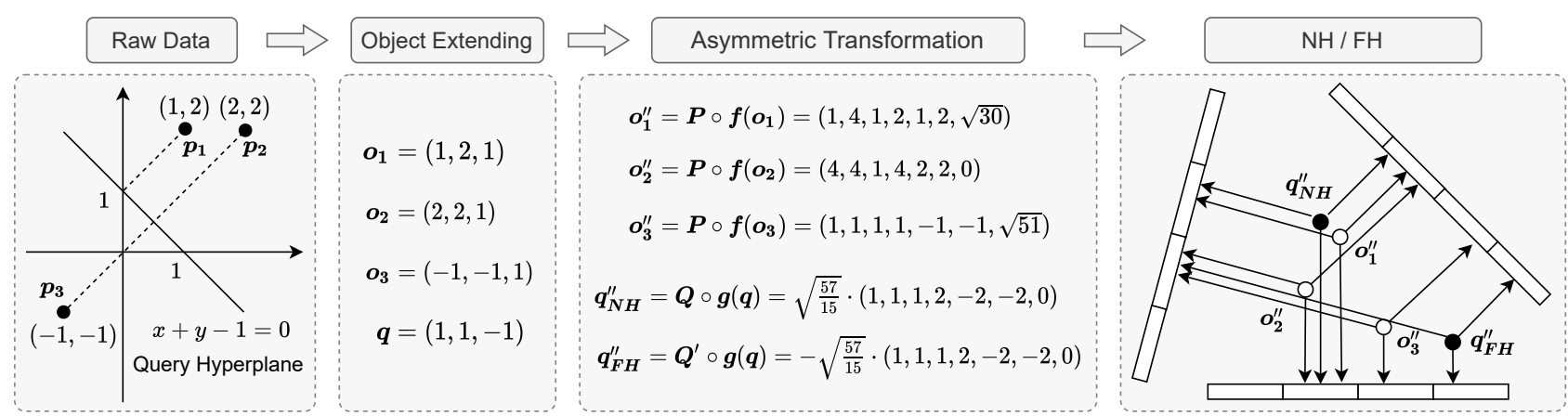

Figure 1: An overview of the hyperplane hashing schemes NH and FH

\subsection{Object Extending}

To simplify Equation 2, we first extend all $\boldsymbol{p} \in \mathcal{D}$ by one dimension with 1, i.e., $\boldsymbol{o}=(\boldsymbol{p} ; 1)=\left(p_{1}, \cdots, p_{d-1}, 1\right)$, where $(;)$ represents the concatenation of dimensions. For a certain $\boldsymbol{q}$, the term $\sqrt{\sum_{i=1}^{d-1} q_{i}^{2}}$ is fixed and is not equal to 0 . For simplicity, we assume $\sqrt{\sum_{i=1}^{d-1} q_{i}^{2}}=1$; otherwise, we can rescale $\boldsymbol{q}$ without changing the order of the P2HNNS results as defined by Equation 1. Then, we have:

$$
d_{P 2 H}(\boldsymbol{p}, \boldsymbol{q})=|\langle\boldsymbol{o}, \boldsymbol{q}\rangle|=\left|\sum_{i=1}^{d} o_{i} q_{i}\right|,
$$

where $\langle\boldsymbol{o}, \boldsymbol{q}\rangle$ is the inner product of $\boldsymbol{o}$ and $\boldsymbol{q}$. Hereafter, we use $\boldsymbol{p} \in \mathbb{R}^{d-1}$ to represent the original data object, $\boldsymbol{o} \in \mathbb{R}^{d}$ to indicate the data object after extending, and $\mathcal{D}^{\prime}$ to denote a database of extended data objects, i.e., $\mathcal{D}^{\prime}=\{\boldsymbol{o} \mid \boldsymbol{o}=(\boldsymbol{p} ; 1), \boldsymbol{p} \in \mathcal{D}\}$.

No LSH and RLSH Families. By converting $d_{P 2 H}(\boldsymbol{p}, \boldsymbol{q})$ into $|\langle\boldsymbol{o}, \boldsymbol{q}\rangle|$, we show it is impossible to construct LSH or RLSH family for $d_{P 2 H}$.

Theorem 3.1: There is no LSH and RLSH families for $d_{P 2 H}(\cdot, \cdot)$.

Proof. We will prove by contradiction. Let $\operatorname{Dist}(\boldsymbol{o}, \boldsymbol{q})=|\langle\boldsymbol{o}, \boldsymbol{q}\rangle|$. For any object $\boldsymbol{o}$ to itself, $\operatorname{Dist}(\boldsymbol{o}, \boldsymbol{o})=|\langle\boldsymbol{o}, \boldsymbol{o}\rangle|=\|\boldsymbol{o}\|^{2}$.

Suppose there exists such an $\left(R, c R, p_{1}, p_{2}\right)$-sensitive LSH family $\mathcal{H}$ for $d_{P 2 H}(\cdot, \cdot)$. We can always construct a data object $\boldsymbol{o}$ such that $0 \leq \operatorname{Dist}(\boldsymbol{o}, \boldsymbol{o})=\|\boldsymbol{o}\|^{2} \leq R$. Then, we can generate a query $\boldsymbol{q}=\frac{2 c R \boldsymbol{o}}{\|\boldsymbol{o}\|^{2}}$ such that $\operatorname{Dist}(\boldsymbol{o}, \boldsymbol{q})=|\langle\boldsymbol{o}, \boldsymbol{q}\rangle|=2 c R \geq c R$ for any $R>0$ and $c>1$. For any $h$ drawn uniformly from $\mathcal{H}$, the collision probability between $\boldsymbol{o}$ and itself is always 1 . Furthermore, according to the definition of LSH family, i.e., Definition 2.1 with the setting $Q(x)=P(x)=x$, the event " $h(\boldsymbol{o})=h(\boldsymbol{q})$ " should have a higher probability than the event " $h(\boldsymbol{o})=h(\boldsymbol{o})$ ", i.e., $\operatorname{Pr}[h(\boldsymbol{o})=h(\boldsymbol{q})]>$ $\operatorname{Pr}[h(\boldsymbol{o})=h(\boldsymbol{o})]=1$. Since any probability cannot be larger than 1 , there is no LSH family for $d_{P 2 H}(\cdot, \cdot)$.

Similarly, we assume there exists an $\left(R, \frac{R}{c}, \xi_{1}, \xi_{2}\right)$-RLSH family $\mathcal{H}$ for $d_{P 2 H}(\cdot, \cdot)$. We can always construct a data object $\boldsymbol{o}$ such that $\operatorname{Dist}(\boldsymbol{o}, \boldsymbol{o})=\|\boldsymbol{o}\|^{2} \geq R$. Then, we can generate a query $\boldsymbol{q}=$ $\frac{R \boldsymbol{o}}{2 c\|\boldsymbol{o}\|^{2}}$ such that $\operatorname{Dist}(\boldsymbol{o}, \boldsymbol{q})=|\langle\boldsymbol{o}, \boldsymbol{q}\rangle|=\frac{R}{2 c} \leq \frac{R}{c}$ for any $R>0$ and $c>1$. For any $h$ drawn uniformly from $\mathcal{H}$, the separation probability between $\boldsymbol{o}$ and itself is always 0 . Furthermore, according to the definition of RLSH family, i.e., Definition 2.2 with the setting $\boldsymbol{Q}(\boldsymbol{x})=\boldsymbol{P}(\boldsymbol{x})=\boldsymbol{x}, \operatorname{Pr}[h(\boldsymbol{o}) \neq h(\boldsymbol{q})]<\operatorname{Pr}[h(\boldsymbol{o}) \neq h(\boldsymbol{o})]=0$. Since any probability cannot be smaller than 0 , there is no RLSH family for $d_{P 2 H}(\cdot, \cdot)$. Therefore, Theorem 3.1 is proved.
Since there is no LSH and RLSH families for $d_{P 2 H}(\cdot, \cdot)$, we follow pioneering works for MIPS and relax the definition of LSH (and RLSH) family by allowing asymmetric transformations (i.e., $P$ and $Q$ ) on data objects and queries. With the use of $\boldsymbol{P}$ and $Q$, the proof of Theorem 3.1 is no longer valid. Thus, there may exist asymmetric LSH and asymmetric RLSH families for $d_{P 2 H}(\cdot, \cdot)$.

\subsection{Asymmetric Transformation}

We now introduce an asymmetric transformation which converts the P2HNNS over $\mathbb{R}^{d}$ into the classic NNS over $\mathbb{R}^{d(d+1) / 2+1}$. It has two steps: object embedding and hypersphere transformation.

Object Embedding. We first design two vector transformations $f: \mathbb{R}^{d} \rightarrow \mathbb{R}^{d(d+1) / 2}$ and $\boldsymbol{g}: \mathbb{R}^{d} \rightarrow \mathbb{R}^{d(d+1) / 2}$ to embed data objects and queries, respectively. Let $\boldsymbol{o}^{\prime}$ and $\boldsymbol{q}^{\prime}$ be the data object and query after object embedding. $f$ and $\boldsymbol{g}$ are defined as follows:

$$
\begin{gathered}
\boldsymbol{o}^{\prime}=\boldsymbol{f}(\boldsymbol{o})=\left(o_{1}^{2}, \cdots, o_{d}^{2}, o_{1} o_{2}, o_{1} o_{3}, \cdots, o_{d-1} o_{d}\right), \\
\boldsymbol{q}^{\prime}=\boldsymbol{g}(\boldsymbol{q})=\left(q_{1}^{2}, \cdots, q_{d}^{2}, 2 q_{1} q_{2}, 2 q_{1} q_{3}, \cdots, 2 q_{d-1} q_{d}\right) .
\end{gathered}
$$

Based on Equations 8 and $9,\left\langle\boldsymbol{o}^{\prime}, \boldsymbol{q}^{\prime}\right\rangle=|\langle\boldsymbol{o}, \boldsymbol{q}\rangle|^{2}$. Thus, with this step, we can remove the absolute value operator appeared in Equation 7.

Hypersphere Transformation. After object embedding, we develop two vector transformations $P: \mathbb{R}^{d(d+1) / 2} \rightarrow \mathbb{R}^{d(d+1) / 2+1}$ and $Q: \mathbb{R}^{d(d+1) / 2} \rightarrow \mathbb{R}^{d(d+1) / 2+1}$ for $\boldsymbol{o}^{\prime}$ and $\boldsymbol{q}^{\prime}$, respectively. Let $M=\max \left\|\boldsymbol{o}^{\prime}\right\| . P$ and $Q$ are defined as follows:

$$
\begin{gathered}
P\left(\boldsymbol{o}^{\prime}\right)=P \circ \boldsymbol{f}(\boldsymbol{o})=\left(\boldsymbol{o}^{\prime} ; \sqrt{M^{2}-\left\|\boldsymbol{o}^{\prime}\right\|^{2}}\right), \\
\boldsymbol{Q}\left(\boldsymbol{q}^{\prime}\right)=Q \circ \boldsymbol{g}(\boldsymbol{q})=\left(-\frac{M}{\left\|\boldsymbol{q}^{\prime}\right\|} \boldsymbol{q}^{\prime} ; 0\right) .
\end{gathered}
$$

As $\left\|\boldsymbol{P}\left(\boldsymbol{o}^{\prime}\right)\right\|=\left\|Q\left(\boldsymbol{q}^{\prime}\right)\right\|=M$, this step maps all $\boldsymbol{o}^{\prime}$ and $\boldsymbol{q}^{\prime}$ to the hypersphere over $\mathbb{R}^{d(d+1) / 2}$ with the same radius $M$. Thus, this step addresses the data normalization problem, and we call it hypersphere transformation. According to Equations 8, 9, 10 and 11,

$$
\|\boldsymbol{P} \circ \boldsymbol{f}(\boldsymbol{o})-Q \circ \boldsymbol{g}(\boldsymbol{q})\|=\sqrt{2 M^{2}+2\langle\boldsymbol{o}, \boldsymbol{q}\rangle^{2}} .
$$

Since $M$ is a fixed constant, according to Equations 7 and 12 ,

$$
\arg \min _{\boldsymbol{o} \in \mathcal{D}^{\prime}}\|\boldsymbol{P} \circ \boldsymbol{f}(\boldsymbol{o})-\boldsymbol{Q} \circ \boldsymbol{g}(\boldsymbol{q})\| \Leftrightarrow \arg \min _{\boldsymbol{p} \in \mathcal{D}} d_{P 2 H}(\boldsymbol{p}, \boldsymbol{q}) .
$$

One can develop different formulas of $\boldsymbol{f}$ and $\boldsymbol{g}$ in object embedding to complete this transformation. For example, following $\mathrm{EH}$ [20], Equation 12 is also satisfied with the formula of $\boldsymbol{f}(\boldsymbol{o})=\boldsymbol{g}(\boldsymbol{q})=$ $\boldsymbol{v e c}\left(\boldsymbol{o o}{ }^{T}\right)$, where $\boldsymbol{v e c}(\cdot)$ vectorizes the rank-1 matrix $\boldsymbol{o o}^{T}$ row by row. However, this design will increase the data dimension from $d$ 
to $d^{2}$, while our proposed $f$ and $g$ increase it from $d$ to $d(d+1) / 2$ only, which can save nearly half of computations.

Randomized Sampling. Our proposed $\boldsymbol{f}$ and $\boldsymbol{g}$, however, still increase the data dimension from $d$ to $O\left(d^{2}\right)$. As will be introduced in Sections 3.4 and 3.5, the hash functions we use contain the inner product $\langle\cdot, \cdot\rangle$, where the computational cost is linear to $d$. Thus, the computation of the hash functions for $P \circ f(o)$ and $Q \circ g(q)$ may be very expensive, especially when $d$ is very large. To alleviate this problem, we follow [20] and use a form of randomized sampling to approximate our proposed asymmetric transformation. This approximation relies on Lemma 3.1 as follows:

Lemma 3.1: Let $\boldsymbol{o}=\left(o_{1}, \cdots, o_{d}\right) \in \mathbb{R}^{d}$ and define $p_{i}=o_{i}^{2} /\|\boldsymbol{o}\|^{2}$. Construct $\tilde{\boldsymbol{o}} \in \mathbb{R}^{d}$ such that the $i^{\text {th }}$ element is $o_{i}$ with probability $p_{i}$ and is 0 otherwise. Select $\lambda$ such elements using sampling with replacement. Then, for any $\boldsymbol{a} \in \mathbb{R}^{d}, \epsilon>0, \tau \geq 1, \lambda \geq \tau / \epsilon^{2}$,

$$
\operatorname{Pr}\left[|\langle\boldsymbol{a}, \tilde{\boldsymbol{o}}\rangle-\langle\boldsymbol{a}, \boldsymbol{o}\rangle| \leq \epsilon\|\boldsymbol{a}\|^{2}\|\boldsymbol{o}\|^{2}\right]>1-\frac{1}{\tau} .
$$

The proof can be referred to the supplementary of [20]. According to Lemma 3.1, when computing the hash value of $P \circ f(o)$ (or $Q \circ \boldsymbol{g}(\boldsymbol{q})$ ), we can first randomly sample the index $i$ of $P \circ f(\boldsymbol{o})$ (or $\boldsymbol{Q} \circ \boldsymbol{g}(\boldsymbol{q})$ ) with probability $p_{i}$ and then compute the hash value through such non-zero indices. This approximation may introduce a small additive error to the hash value, but the computing time can be reduced from $O\left(d^{2}\right)$ to $O\left(1 / \epsilon^{2}\right)$ for any $\epsilon>0$.

Until now, we have presented the asymmetric transformation and discussed its approximation with randomized sampling. Next, we will introduce the details of $\mathrm{NH}$ and $\mathrm{FH}$ for P2HNNS.

\subsection{Nearest Hyperplane Hashing}

NH Hash Family. Let $h_{\boldsymbol{a}, b}(\cdot)$ be a random projection LSH function as defined by Equation 3 . Given the asymmetric transformation $\boldsymbol{P} \circ \boldsymbol{f}(\boldsymbol{o})$ and $Q \circ \boldsymbol{g}(\boldsymbol{q})$, the NH hash family $\mathcal{H}_{\mathcal{N}}$ of hash functions $h_{\mathcal{N}}(\cdot)$ is defined as follows:

$$
h_{\mathcal{N}}(x)= \begin{cases}h_{\boldsymbol{a}, b}(\boldsymbol{P} \circ f(x)), & \text { if } x \text { is a data object } \boldsymbol{o}, \\ h_{\boldsymbol{a}, b}(\boldsymbol{Q} \circ \boldsymbol{g}(\boldsymbol{x})), & \text { if } x \text { is a hyperplane query } \boldsymbol{q} .\end{cases}
$$

According to Equations 7 and 12, the collision probability of $h_{\mathcal{N}}(\cdot)$ for certain $\boldsymbol{o}, \boldsymbol{q}$ is computed as follows:

$$
\operatorname{Pr}\left[h_{\mathcal{N}}(\boldsymbol{o})=h_{\mathcal{N}}(\boldsymbol{q})\right]=p\left(\sqrt{2 M^{2}+2\langle\boldsymbol{o}, \boldsymbol{q}\rangle^{2}}\right),
$$

where $p(\cdot)$ is defined by Equation 4 . Let $p_{1}=p\left(\sqrt{2 M^{2}+2 R^{2}}\right)$ and $p_{2}=p\left(\sqrt{2 M^{2}+2 c^{2} R^{2}}\right)$. With $\boldsymbol{P} \circ \boldsymbol{f}(\boldsymbol{o})$ and $\boldsymbol{Q} \circ \boldsymbol{g}(\boldsymbol{q})$, we show $\mathcal{H}_{\mathcal{N}}$ is an asymmetric LSH family for $d_{P 2 H}(\cdot, \cdot)$.

Lemma 3.2: Given a search radius $R(R>0)$, an approximation ratio $c(c>1)$, and $a\left(\sqrt{2 M^{2}+2 R^{2}}, \sqrt{2 M^{2}+2 c^{2} R^{2}}, p_{1}, p_{2}\right)$-sensitive hash family $\mathcal{H}$ for Euclidean distance for NNS, the NH hash family $\mathcal{H}_{\mathcal{N}}$ of hash functions $h_{\mathcal{N}}(\cdot)$ is $\left(R, c R, p_{1}, p_{2}\right)$-sensitive for $d_{P 2 H}(\cdot, \cdot)$.

Proof. According to Equations 7 and 12, we have:

$$
\begin{gathered}
d_{P 2 H}(\boldsymbol{p}, \boldsymbol{q}) \leq R \Leftrightarrow\|\boldsymbol{P} \circ \boldsymbol{f}(\boldsymbol{o})-\boldsymbol{Q} \circ \boldsymbol{g}(\boldsymbol{q})\| \leq \sqrt{2 M^{2}+2 R^{2}}, \\
d_{P 2 H}(\boldsymbol{p}, \boldsymbol{q}) \geq c R \Leftrightarrow\|\boldsymbol{P} \circ \boldsymbol{f}(\boldsymbol{o})-\boldsymbol{Q} \circ \boldsymbol{g}(\boldsymbol{q})\| \geq \sqrt{2 M^{2}+2 c^{2} R^{2}} .
\end{gathered}
$$

Recall that $p_{1}=p\left(\sqrt{2 M^{2}+2 R^{2}}\right)$ and $p_{2}=p\left(\sqrt{2 M^{2}+2 c^{2} R^{2}}\right)$. Since $\mathcal{H}$ is a $\left(\sqrt{2 M^{2}+2 R^{2}}, \sqrt{2 M^{2}+2 c^{2} R^{2}}, p_{1}, p_{2}\right)$-sensitive hash

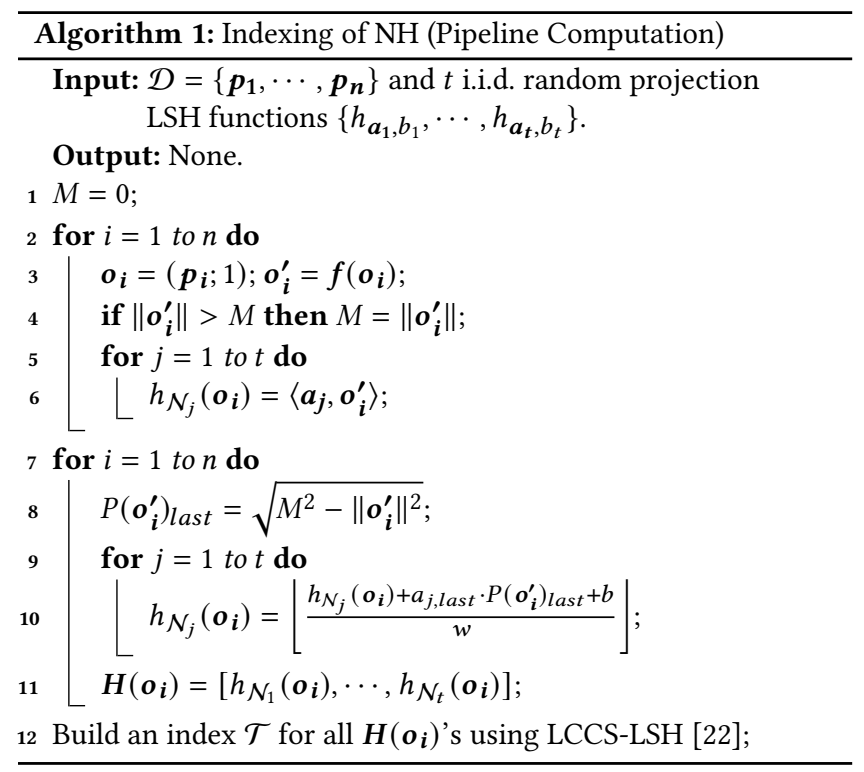

family for Euclidean distance for NNS, based on Expression 14 and Definition 2.1, we infer that

$$
\begin{aligned}
d_{P 2 H}(\boldsymbol{p}, \boldsymbol{q}) \leq R & \Rightarrow \operatorname{Pr}\left[h_{\mathcal{N}}(\boldsymbol{o})=h_{\mathcal{N}}(\boldsymbol{q})\right] \geq p_{1}, \\
d_{P 2 H}(\boldsymbol{p}, \boldsymbol{q}) \geq c R & \Rightarrow \operatorname{Pr}\left[h_{\mathcal{N}}(\boldsymbol{o})=h_{\mathcal{N}}(\boldsymbol{q})\right] \leq p_{2} .
\end{aligned}
$$

Since $R>0$ and $c>1, p_{1}>p_{2}$. Thus, the NH hash family $\mathcal{H}_{\mathcal{N}}$ of hash functions $h_{\mathcal{N}}(\cdot)$ is $\left(R, c R, p_{1}, p_{2}\right)$-sensitive for $d_{P 2 H}(\cdot, \cdot)$.

NH. With the NH hash family $\mathcal{H}_{\mathcal{N}}$, we describe NH for P2HNNS. $\mathrm{NH}$ consists of two phases: indexing phase and query phase.

Indexing Phase. We propose a pipeline computation to build index. Specifically, once we extend each $\boldsymbol{p}$ to $\boldsymbol{o}$ and embed $\boldsymbol{o}$ to $\boldsymbol{f}(\boldsymbol{o})$, we immediately compute the intermediate value of $h_{\mathcal{N}}(o)$ for $f(o)$. According to Equation 10, we require a global $M$ to compute $\boldsymbol{P}\left(\boldsymbol{o}^{\prime}\right)$. However, since $h_{\mathcal{N}}(\boldsymbol{o})$ contains the inner product $\langle\boldsymbol{a}, \boldsymbol{P} \circ \boldsymbol{f}(\boldsymbol{o})\rangle$ which is a linear operation of cumulative addition, we can postpone the addition of the last element $\sqrt{M^{2}-\left\|\boldsymbol{o}^{\prime}\right\|^{2}}$ of $\boldsymbol{P}\left(\boldsymbol{o}^{\prime}\right)$ until we determine the $M$ value. We repeat this procedure $t$ times to get $t$ $h_{\mathcal{N}_{j}}(\boldsymbol{o})$ 's. Let $\boldsymbol{H}(\boldsymbol{o})=\left[h_{\mathcal{N}_{1}}(\boldsymbol{o}), \cdots, h_{\mathcal{N}_{t}}(\boldsymbol{o})\right]$ be the hash string of $\boldsymbol{o}$. Finally, $\mathrm{NH}$ adopts LCCS-LSH to build an index $\mathcal{T}$ for all $\boldsymbol{H}(\boldsymbol{o})$ 's. The pseudo-code is depicted in Algorithm 1.

This pipeline computation can avoid $O\left(n d^{2}\right)$ space to store the intermediate values $\boldsymbol{P} \circ \boldsymbol{f}(\boldsymbol{o})$ for all $\boldsymbol{o}$ 's. Moreover, with the linear property of $\langle\cdot, \cdot\rangle$, it will not introduce extra error for $h_{\mathcal{N}}(\boldsymbol{o})$.

Query Phase. Given a P2HNNS query $\boldsymbol{q}$, NH first computes its hash $\overline{\text { string } \boldsymbol{H}(\boldsymbol{q})}=\left[h_{\mathcal{N}_{1}}(\boldsymbol{q}), \cdots, h_{\mathcal{N}_{t}}(\boldsymbol{q})\right]$. Then, it calls LCCS-LSH for NNS to identify a set of candidates $C$ with $\mathcal{T}$. Finally, NH determines the nearest point $\boldsymbol{p}_{\min }$ from $C$ as the final answer. The pseudo-code of $\mathrm{NH}$ for the P2HNNS is shown in Algorithm 2.

Example 3.1: We now use Figure 1 again to illustrate how to conduct the P2HNNS with NH. Suppose $d=3, \mathcal{D}=\left\{\boldsymbol{p}_{1}, \boldsymbol{p}_{2}, \boldsymbol{p}_{3}\right\}$, and there exists a hyperplane query $\boldsymbol{q}=(1,1,-1)$.

First of all, we extend each $\boldsymbol{p}_{\boldsymbol{i}}$ by one more dimension with 1 , e.g., $\boldsymbol{o}_{1}=\left(\boldsymbol{p}_{1} ; 1\right)=(1,2,1)$, so that all $\boldsymbol{o}_{\boldsymbol{i}}$ 's and $\boldsymbol{q}$ are in the same 


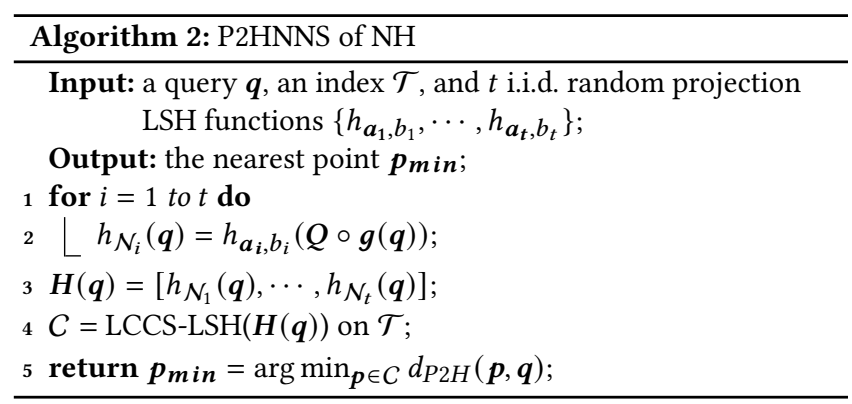

dimension. Then, we use $\boldsymbol{f}$ and $\boldsymbol{g}$ to embed all $\boldsymbol{o}_{\boldsymbol{i}}$ and $\boldsymbol{q}$ from $\mathbb{R}^{3}$ to $\mathbb{R}^{6}$, e.g., according to Equations 8 and $9, \boldsymbol{o}_{1}^{\prime}=\boldsymbol{f}\left(\boldsymbol{o}_{1}\right)=(1,4,1,2,1,2)$, $\boldsymbol{q}^{\prime}=\boldsymbol{g}(\boldsymbol{q})=(1,1,1,2,-2,-2)$, and $\left\langle\boldsymbol{o}_{1}^{\prime}, \boldsymbol{q}^{\prime}\right\rangle=\left|\left\langle\boldsymbol{o}_{1}, \boldsymbol{q}\right\rangle\right|^{2}=4$. After the step of object embedding, we get $M=\max \left\|o_{i}^{\prime}\right\|=\sqrt{57}$. Next, we use $\boldsymbol{P}$ and $\boldsymbol{Q}$ to convert all $\boldsymbol{o}_{\boldsymbol{i}}^{\prime}$ and $\boldsymbol{q}^{\prime}$ from $\mathbb{R}^{6}$ to $\mathbb{R}^{7}$. For example, according to Equations 10 and $11, \boldsymbol{o}_{1}^{\prime \prime}=\boldsymbol{P}\left(\boldsymbol{o}_{1}^{\prime}\right)=(1,4,1,2,1,2, \sqrt{30})$, $\boldsymbol{q}_{\boldsymbol{N} \boldsymbol{H}}^{\prime \prime}=Q\left(\boldsymbol{q}^{\prime}\right)=-\sqrt{\frac{57}{15}}(1,1,1,2,-2,-2,0)$, and we have $\left\|\boldsymbol{o}_{\boldsymbol{i}}^{\prime \prime}\right\|=$ $\left\|\boldsymbol{q}_{N H}^{\prime \prime}\right\|=\sqrt{57}$. With this asymmetric transformation, the P2HNNS over $\mathbb{R}^{3}$ are converted into the NNS over $\mathbb{R}^{7}$. Finally, NH leverages LCCS-LSH to find the nearest neighbor of $\boldsymbol{q}_{\boldsymbol{N} H}^{\prime \prime}$. Let $t=3$. We can see that $\boldsymbol{o}_{1}^{\prime \prime}$ collides with $\boldsymbol{q}_{\boldsymbol{N} H}^{\prime \prime}$ in 3 times, which is higher than $\boldsymbol{o}_{2}^{\prime \prime}$ and $\boldsymbol{o}_{3}^{\prime \prime}$. Since $\boldsymbol{o}_{1}^{\prime \prime}$ has the longest common hash strings with $q_{N H}^{\prime \prime}$, $\mathrm{NH}$ returns $\boldsymbol{p}_{1}$ as the answer.

Based on Theorem 2.1 and Lemma 3.2, we show NH can deal with the P2HNNS in sub-quadratic space and sub-linear query time.

Theorem 3.2: Given a family $\mathcal{H}_{\mathcal{N}}$ of hash functions $h_{\mathcal{N}}(\cdot)$ as defined by Equation 13, setting $t=O\left(n^{\rho}\right), N H$ is a data structure which can find a $\boldsymbol{p} \in \mathcal{D}$ such that $d_{P 2 H}(\boldsymbol{p}, \boldsymbol{q}) \leq c \cdot d_{P 2 H}\left(\boldsymbol{p}^{*}, \boldsymbol{q}\right)$ with constant probability in $O\left(n^{1+\rho}\right)$ space, $O\left(n^{1+\rho}\left(d^{2}+\log n\right)\right)$ indexing time, and $O\left(n^{\rho}\left(d^{2}+\log n\right)\right)$ query time, where $\rho=\ln \left(1 / p_{1}\right) / \ln \left(1 / p_{2}\right)$ and $\boldsymbol{p}^{*}=\arg \min _{\boldsymbol{p} \in \mathcal{D}} d_{P 2 H}(\boldsymbol{p}, \boldsymbol{q})$.

According to Theorem 3.2, NH has an accuracy guarantee on $c$ for P2HNNS, but for the NNS, the accuracy guarantee $c^{\prime}$ of LCCS-LSH is not equal to $c$. According to Lemma 3.2, we require a $\left(\sqrt{2 M^{2}+2 R^{2}}\right.$, $\left.\sqrt{2 M^{2}+2 c^{2} R^{2}}, p_{1}, p_{2}\right)$-sensitive hash family for NNS. Thus, the accuracy guarantee of LCCS-LSH is $c^{\prime}=\sqrt{2 M^{2}+2 c^{2} R^{2}} / \sqrt{2 M^{2}+2 R^{2}}$. Moreover, from Theorem 3.2, $\boldsymbol{P} \circ \boldsymbol{f}(\boldsymbol{o})$ and $\boldsymbol{Q} \circ \boldsymbol{g}(\boldsymbol{q})$ will increase the computational cost of $h_{\mathcal{N}}(\cdot)$ from $d$ to $O\left(d^{2}\right)$, leading to large indexing and query time complexities of $\mathrm{NH}$. In practice, we will adopt the randomized sampling as discussed in Section 3.3 for $\mathrm{NH}$ to reduce this computational cost.

\subsection{Furthest Hyperplane Hashing}

Accroding to Equation 14, there exists a large constant $2 M^{2}$ when we compute the collision probability for the $\mathrm{NH}$ hash family. It will diminish the difference between $p_{1}$ and $p_{2}$, and hence the close data objects might be hard to be identified from the far-apart ones. Similar to $\mathrm{EH}, \mathrm{BH}$, and $\mathrm{MH}$, when $d_{P 2 H}(\boldsymbol{p}, \boldsymbol{q}) \rightarrow 0, \lim _{d_{P 2 H} \rightarrow 0} p_{1}=$ $\lim _{d_{P 2 H} \rightarrow 0} p_{2}=p(\sqrt{2} M)$, the $\rho$ value of $\mathrm{NH}$ will approach to 1 , i.e., $\rho=\lim _{d_{P 2 H} \rightarrow 0} \ln \left(1 / p_{1}\right) / \ln \left(1 / p_{2}\right)=1$. Thus, we consider to convert the problem of P2HNNS into another classic problem of point-to-point FNS and present FH accordingly.
New Variant of Asymmetric Transformation. The new variant of asymmetric transformation follows the same step of object embedding $f$ and $g$, but we define a new hypersphere transformation of $Q^{\prime}$ for $\boldsymbol{q}^{\prime}$ :

$$
Q^{\prime}\left(\boldsymbol{q}^{\prime}\right)=Q^{\prime} \circ \boldsymbol{g}(\boldsymbol{q})=\left(\frac{M}{\left\|\boldsymbol{q}^{\prime}\right\|} \boldsymbol{q}^{\prime} ; 0\right) .
$$

According to Equations 8, 9, 10 and 15, we have:

$$
\left\|\boldsymbol{P} \circ \boldsymbol{f}(\boldsymbol{o})-Q^{\prime} \circ \boldsymbol{g}(\boldsymbol{q})\right\|=\sqrt{2 M^{2}-2\langle\boldsymbol{o}, \boldsymbol{q}\rangle^{2}} .
$$

According to Equations 7 and 16, we have:

$$
\arg \max _{\boldsymbol{o} \in \mathcal{D}^{\prime}}\left\|\boldsymbol{P} \circ \boldsymbol{f}(\boldsymbol{o})-Q^{\prime} \circ \boldsymbol{g}(\boldsymbol{q})\right\| \Leftrightarrow \arg \min _{\boldsymbol{p} \in \mathcal{D}} d_{P 2 H}(\boldsymbol{p}, \boldsymbol{q}) .
$$

Thus, with $\boldsymbol{P} \circ \boldsymbol{f}(\boldsymbol{o})$ and $Q^{\prime} \circ \boldsymbol{g}(\boldsymbol{q})$, the P2HNNS over $\mathbb{R}^{d}$ can be reduced to the classic FNS over $\mathbb{R}^{d(d+1) / 2+1}$. As will be shown in Equation 18, by making $\left(2 M^{2}-2\langle\boldsymbol{o}, \boldsymbol{q}\rangle^{2}\right)$, this new asymmetric transformation can amplify the difference between $\xi_{1}$ and $\xi_{2}$.

FH Hash Family. Suppose $h_{\boldsymbol{a}}(\cdot)$ is an RQALSH function as defined by Equation 5 . Given this new variant of asymmetric transformation, we define an FH hash family $\mathcal{H}_{\mathcal{F}}$ of hash functions $h_{\mathcal{F}}(\cdot)$ as follows:

$$
h_{\mathcal{F}}(x)= \begin{cases}h_{\boldsymbol{a}}(P \circ f(x)), & \text { if } x \text { is a data object } o, \\ h_{\boldsymbol{a}}\left(Q^{\prime} \circ g(x)\right), & \text { if } x \text { is a hyperplane query } \boldsymbol{q} .\end{cases}
$$

According to Equations 7 and 16, the separation probability of $h_{\mathcal{F}}(\cdot)$ for certain $\boldsymbol{o}, \boldsymbol{q}$ is computed as follows:

$$
\operatorname{Pr}\left[\left|h_{\mathcal{F}}(\boldsymbol{o})-h_{\mathcal{F}}(\boldsymbol{q})\right|>\frac{w}{2}\right]=\xi\left(\sqrt{2 M^{2}-2\langle\boldsymbol{o}, \boldsymbol{q}\rangle^{2}}\right),
$$

where $\xi(\cdot)$ is defined by Equation 6 . Let $\xi_{1}=\xi\left(\sqrt{2 M^{2}-2 R^{2} / c^{2}}\right)$ and $\xi_{2}=\xi\left(\sqrt{2 M^{2}-2 R^{2}}\right)$. With this new variant of asymmetric transformation, we show $\mathcal{H}_{\mathcal{F}}$ is an asymmetric RLSH family for $d_{P 2 H}(\cdot, \cdot)$.

Lemma 3.3: Given a search radius $R(R>0)$, an approximation ratioc $(c>1)$, and $a\left(\sqrt{2 M^{2}-2 R^{2} / c^{2}}, \sqrt{2 M^{2}-2 R^{2}}, \xi_{1}, \xi_{2}\right)$-sensitive hash family for Euclidean distance for FNS, the FH hash family $\mathcal{H}_{\mathcal{F}}$ of hash functions $h_{\mathcal{F}}(\cdot)$ is $\left(\frac{R}{c}, R, \xi_{1}, \xi_{2}\right)$-sensitive for $d_{P 2 H}(\cdot, \cdot)$.

Proof. The proof of Lemma 3.3 is similar to that of Lemma 3.2. To be concise, we omit the details here.

Trivial FH. With the FH hash family $\mathcal{H}_{\mathcal{F}}$, we first introduce a trivial version of $\mathrm{FH}$ for $\mathrm{P} 2 \mathrm{HNNS}$. In the indexing phase, the trivial FH first generates $m$ i.i.d. RQALSH functions as defined by Equation 6. Then, it extends each $\boldsymbol{p}$ to $\boldsymbol{o}$ and follows the pipeline computation of NH to calculate $h_{\mathcal{F}_{1}}(\boldsymbol{o}), \cdots, h_{\mathcal{F}_{m}}(\boldsymbol{o})$ for each $\boldsymbol{o}$. Finally, it uses RQALSH to build index with the hash values of $n$ data objects. In the query phase, after computing $h_{\mathcal{F}_{1}}(\boldsymbol{q}), \cdots, h_{\mathcal{F}_{m}}(\boldsymbol{q})$, the trivial FH uses RQALSH to identify a set of candidates $C$ for the FNS of $Q^{\prime} \circ \boldsymbol{g}(\boldsymbol{q})$ whose separation countings are more than $l$ times. It returns $\boldsymbol{p}_{\min }=\arg \min _{\boldsymbol{p} \in C} d_{P 2 H}(\boldsymbol{p}, \boldsymbol{q})$ as the final answer.

We choose RQALSH for FNS based on two considerations: (1) it is a competitive method that has been shown to be superior to other state-of-the-art hashing schemes, such as QDAFN [30] and DrusillaSelect [10]; (2) it is independent of $d$ and enjoys quality guarantee on query results, which helps us establish quality guarantee for $\mathrm{FH}$. Based on Theorem 2.2 and Lemma 3.3, we show that the trivial FH enjoys a theoretical guarantee for P2HNNS. 
Theorem 3.3: Given a family $\mathcal{H}_{\mathcal{F}}$ of hash functions $h_{\mathcal{F}}(\cdot)$ as defined by Equation 17, setting $m=O(\log n)$ and $l=O(\log n)(l \leq m)$, the trivial $F H$ is a data structure which can find a $p \in \mathcal{D}$ such that $d_{P 2 H}(\boldsymbol{p}, \boldsymbol{q}) \leq c \cdot d_{P 2 H}\left(\boldsymbol{p}^{*}, \boldsymbol{q}\right)$ with constant probability in $O(n \log n)$ space, $O\left(\left(d^{2}+\log n\right) n \log n\right)$ indexing time, and $O\left(\left(d^{2}+n\right) \log n\right)$ query time, where $\boldsymbol{p}^{*}=\arg \min _{\boldsymbol{p} \in \mathcal{D}} d_{P 2 H}(\boldsymbol{p}, \boldsymbol{q})$.

Similar to Theorem 3.2, we can find that the trivial FH has an accuracy guarantee on $c$ for P2HNNS, but for the FNS, the accuracy guarantee $c^{\prime \prime}$ of RQALSH is not equal to $c$. According to Lemma 3.3, we need a $\left(\sqrt{2 M^{2}-2 R^{2} / c^{2}}, \sqrt{2 M^{2}-2 R^{2}}, \xi_{1}, \xi_{2}\right)$-sensitive hash family for FNS. Thus, the accuracy guarantee of RQALSH is $c^{\prime \prime}=$ $\sqrt{2 M^{2}-2 R^{2} / c^{2}} / \sqrt{2 M^{2}-2 R^{2}}$.

$\underline{\text { Remarks. }}$ Compared with NH, the trivial FH enjoys less space and indexing time complexities, but its query time complexity is also much worse than that of $\mathrm{NH}$. However, it is analysed in the worst case, in the sense that RQALSH conducts separation counting for all $n$ data objects in all $m$ hash tables. For the real-life datasets in high-dimensional spaces, data points are sparse and often lie on a low-dimensional manifold. As such, most data points do not need to be checked for all $m$ hash tables. The query time of the trivial $\mathrm{FH}$ in practice may be better than the theoretical bound.

However, once $\boldsymbol{o}$ satisfies $\left|h_{\mathcal{F}}(\boldsymbol{o})-h_{\mathcal{F}}(\boldsymbol{q})\right|>\frac{w}{2}$, RQALSH must conduct a separation counting for $\boldsymbol{o}$, no matter whether $d_{P 2 H}(\boldsymbol{o}, \boldsymbol{q})$ is small or large. Among the $m$ hash tables, RQALSH may require checking all $n$ data objects with at least once. Thus, in average case, the trivial FH still requires $O\left(n+d^{2} \log n\right)$ query time for P2HNNS.

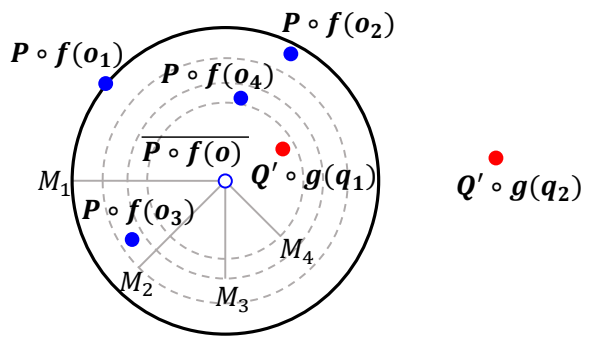

Figure 2: An example of the data-dependent multi-partition strategy

FH with a Data-Dependent Multi-Partition Strategy. As $n$ increases, the performance of the trivial FH may degrade rapidly, because it requires conducting pointless separation counting for more and more false positives. With the new $\boldsymbol{P} \circ \boldsymbol{f}(\boldsymbol{o})$ and $Q^{\prime} \circ \boldsymbol{g}(\boldsymbol{q})$, we now introduce a data-dependent multi-partition strategy to speed up the trivial FH. Referring to Figure 2, let $\overline{\boldsymbol{P} \circ \boldsymbol{f ( o )}}$ be the centroid of all data objects after $\boldsymbol{P} \circ \boldsymbol{f}(\cdot)$, i.e., $\overline{\boldsymbol{P} \circ \boldsymbol{f ( \boldsymbol { o } )}}=\frac{1}{n} \sum_{i=1}^{n} \boldsymbol{P} \circ \boldsymbol{f}\left(\boldsymbol{o}_{\boldsymbol{i}}\right)$. No matter for $Q^{\prime} \circ g\left(q_{1}\right)$ which is close to $\overline{P \circ f(o)}$ or for $Q^{\prime} \circ g\left(q_{2}\right)$ which is far from $\overline{P \circ f(o)}$, the data objects further from $\overline{P \circ f(o)}$ are more likely to be the furthest neighbors of $Q^{\prime} \circ g(q)$.

Indexing Phase. Based on the above observation, in the indexing phase, we first extend each $\boldsymbol{p}_{\boldsymbol{i}}$ to $\boldsymbol{o}_{\boldsymbol{i}}$. Then, we compute the centroid $\overline{P \circ f(o)}$ of $n$ data objects after $P \circ f(\cdot)$ and sort them in descending order of $\left\|\boldsymbol{P} \circ \boldsymbol{f}\left(\boldsymbol{o}_{\boldsymbol{i}}\right)-\overline{\boldsymbol{P} \circ \boldsymbol{f}(\boldsymbol{o})}\right\|$. Let $\sigma$ be the interval ratio $(0<$ $\sigma<1)$. We follow that order and partition each $\boldsymbol{o}_{\boldsymbol{i}}$ into $T$ disjoint subsets $\left\{\mathcal{S}_{1}, \cdots, \mathcal{S}_{T}\right\}$ such that $\sigma M_{j}<\left\|\boldsymbol{P} \circ \boldsymbol{f}\left(\boldsymbol{o}_{\boldsymbol{i}}\right)-\overline{\boldsymbol{P} \circ \boldsymbol{f}(\boldsymbol{o})}\right\| \leq M_{j}$
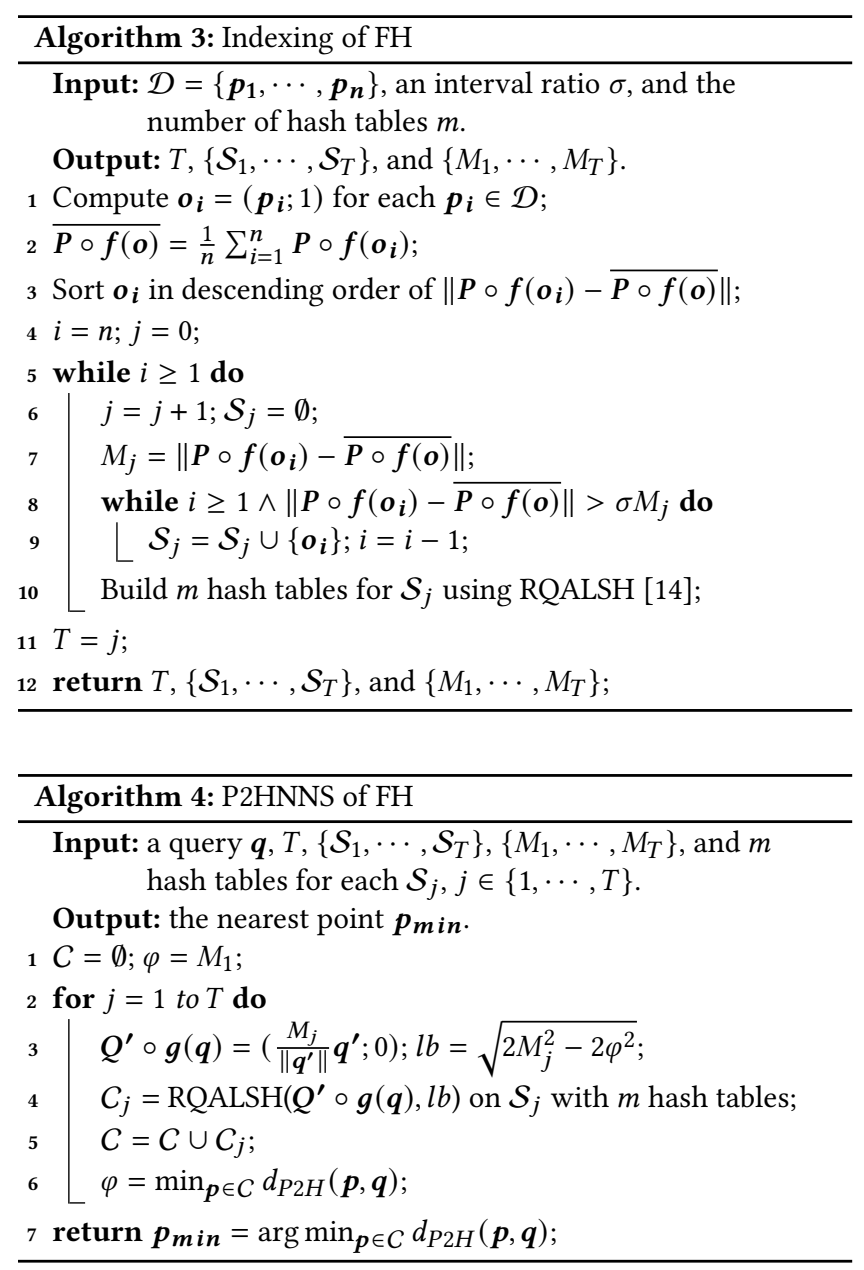

for $\boldsymbol{o}_{\boldsymbol{i}} \in \mathcal{S}_{j}$, where $M_{j}=\max _{\boldsymbol{o}_{\boldsymbol{i}} \in \mathcal{S}_{j}}\left\|\boldsymbol{P} \circ \boldsymbol{f}\left(\boldsymbol{o}_{\boldsymbol{i}}\right)-\overline{\boldsymbol{P} \circ \boldsymbol{f}(\boldsymbol{o})}\right\|$ and $j \in\{1, \cdots, T\}$. Finally, we follow the trivial $\mathrm{FH}$ and use RQALSH to build $m$ hash tables individually for each $\mathcal{S}_{j}$. The pseudo-code of the indexing of $\mathrm{FH}$ is depicted in Algorithm 3.

Note that $M_{1}, \cdots, M_{T}$ are sorted in descending order. The number $T$ of disjoint subsets is automatically determined by the interval ratio $\sigma$ and the data distribution after $P \circ f(\cdot)$. Thus, we call this strategy as a data-dependent multi-partition strategy.

Query Phase. Given a P2HNNS query $\boldsymbol{q}$, FH uses RQALSH to identify a set of candidates $C$ for the FNS of $Q^{\prime} \circ \boldsymbol{g}(\boldsymbol{q})$ from $\mathcal{S}_{1}$ to $\mathcal{S}_{T}$. Let $\varphi$ be the smallest $d_{P 2 H}(\boldsymbol{p}, \boldsymbol{q})$ we find so far, i.e., $\varphi=\min _{\boldsymbol{p} \in C} d_{P 2 H}(\boldsymbol{p}, \boldsymbol{q})$. With $M_{j}$ and $\varphi$, we compute the lower bound $l b$ for the FNS, i.e., $l b=\sqrt{2 M_{j}^{2}-2 \varphi^{2}}$. When using RQALSH for FNS on $\mathcal{S}_{j}$, we can stop early if $\left\|\boldsymbol{P} \circ \boldsymbol{f}\left(\boldsymbol{o}_{\boldsymbol{i}}\right)-Q^{\prime} \circ \boldsymbol{g}(\boldsymbol{q})\right\| \leq l b$. Finally, FH determines $p_{\text {min }}=\arg \min _{\boldsymbol{p} \in C} d_{P 2 H}(\boldsymbol{p}, \boldsymbol{q})$ as the final answer. The pseudocode of FH for P2HNNS is shown in Algorithm 4.

As we first search the data objects far-away from $\overline{P \circ f(o)}$, which probably contains the furthest neighbors of $Q^{\prime} \circ \boldsymbol{g}(\boldsymbol{q})$, the search process might be stopped in the first few rounds of $\mathcal{S}_{j}$, so we can avoid most unnecessary separation counting for the false positives. Therefore, FH can be much faster than the trivial FH. 


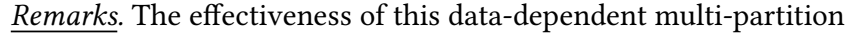
strategy, however, is highly sensitive to the data distribution. If most data objects after $P \circ f(\cdot)$ are close to $\overline{P \circ f(o)}$, i.e., $\| P \circ f\left(o_{i}\right)-$ $\overline{\boldsymbol{P} \circ \boldsymbol{f ( o )} \|} \ll M_{1}$, then our strategy can reduce separation counting for a large number of false positives and boost the search efficiency of FH significantly. Nevertheless, if most data objects after $P \circ f(\cdot)$ are far from $\overline{\boldsymbol{P} \circ \boldsymbol{f ( o )}}$, i.e., $\left\|\boldsymbol{P} \circ \boldsymbol{f}\left(\boldsymbol{o}_{\boldsymbol{i}}\right)-\overline{\boldsymbol{P} \circ \boldsymbol{f ( o )}}\right\| \gg 0$, our strategy is not effective because RQALSH requires conducting separation counting for many false positives. In the worst case, all of the data objects fall in $\mathcal{S}_{1}$, i.e., $\sigma M_{1}<\left\|\boldsymbol{P} \circ \boldsymbol{f}\left(\boldsymbol{o}_{\boldsymbol{i}}\right)-\overline{\boldsymbol{P} \circ \boldsymbol{f}(\boldsymbol{o})}\right\| \leq M_{1}$ for all $\boldsymbol{o}_{\boldsymbol{i}}$ 's. Then, FH will decline to its trivial version.

Based on Theorems 2.2 and 3.3, we have Corollary 3.1 as follows:

Corollary 3.1: FH with the data-dependent multi-partition strategy is a data structure which can find a $\boldsymbol{p} \in \mathcal{D}$ such that $d_{P 2 H}(\boldsymbol{p}, \boldsymbol{q}) \leq$ $c \cdot d_{P 2 H}\left(\boldsymbol{p}^{*}, \boldsymbol{q}\right)$ with constant probability in $O(n \log n)$ space, $O\left(\left(d^{2}+\right.\right.$ $\log n) n \log n)$ indexing time, and $O\left(\left(d^{2}+n\right) \log n\right)$ query time, where $\boldsymbol{p}^{*}=\arg \min _{\boldsymbol{p} \in \mathcal{D}} d_{P 2 H}(\boldsymbol{p}, \boldsymbol{q})$.

Similar to NH, the asymmetric transformation will increase the computational cost of $\mathrm{FH}$ hash functions from $d$ to $O\left(d^{2}\right)$, leading to large indexing and query time complexities. Thus, we will also adopt the randomized sampling for FH to alleviate this problem.

\section{EXPERIMENTS}

We study the performance of $\mathrm{NH}$ and $\mathrm{FH}$ over five real-life datasets for high-dimensional P2HNNS. All methods are implemented in $\mathrm{C}++$ and compiled with $\mathrm{g}++8.3 \mathrm{using}-\mathrm{O} 3$ optimization. We conduct all experiments in a single thread on a machine with 8 Intel i7-3820 @ $3.60 \mathrm{GHz}$ CPUs and 64 GB RAM, running on Ubuntu 18.04 .

\subsection{Datasets and Queries}

We use five real-life datasets Yelp, ${ }^{1}$ Music-100, ${ }^{2}$ GloVe,${ }^{3}$ Tiny-1M [40], and Msong ${ }^{4}$ in our experiments, which cover a wide range of data types, including rating, text, image, and audio data. We randomly generate 100 hyperplane queries for each dataset. The details of the hyperplane query generation can be referred to Appendix A The statistics of datasets and queries are summarized in Table 1.

\subsection{Evaluation Metrics}

We use the following metrics for performance evaluation.

- Index Size and Indexing Time. We use the index size and indexing time to evaluate the indexing overhead of a method. The index size is defined by the memory usage for a method to build index. Similarly, the indexing time is defined as the wall-clock time for a method to build index.

- Recall. We use recall to measure the accuracy of a method. For the $k$-P2HNNS, it is defined as the fraction of the total amount data objects returned by a method that are appeared in the exact $k$ nearest objects to the hyperplane query.

- Query Time. We consider the query time to evaluate the efficiency of a method. It is defined as the wall-clock time of a method to conduct the $k$-P2HNNS.

\footnotetext{
${ }^{1}$ Round 7 of Yelp Data Challenge: http://www.yelp.com/dataset_challenge.

${ }^{2}$ Music-100: https://github.com/stanis-morozov/ip-nsw.

${ }^{3}$ GloVe: https://nlp.stanford.edu/projects/glove/.

${ }^{4}$ Msong: http://www.ifs.tuwien.ac.at/mir/msd/download.html.
}

Table 1: Statistics of datasets and queries

\begin{tabular}{cccccc}
\hline Datasets & $n$ & $d$ & \#Queries & Data Size & Type \\
\hline Yelp & 77,079 & 50 & 100 & $14.7 \mathrm{MB}$ & Rating \\
Music-100 & $1,000,000$ & 100 & 100 & $381.5 \mathrm{MB}$ & Rating \\
GloVe & $1,183,514$ & 100 & 100 & $451.5 \mathrm{MB}$ & Text \\
Tiny-1M & $1,000,000$ & 384 & 100 & $1.43 \mathrm{~GB}$ & Image \\
Msong & 992,272 & 420 & 100 & $1.55 \mathrm{~GB}$ & Audio \\
\hline
\end{tabular}

- Fraction. We also use the fraction to access the efficiency of a method. It is defined as the fraction of the number of candidates verified by a method over the $n$ data objects.

We report the average recall and fraction over all queries, and we run each method for each experiment five times to report its average query time and indexing overhead.

\subsection{Benchmark Methods}

Both $\mathrm{NH}$ and $\mathrm{FH}$ are designed for P2HNNS beyond the unit hypersphere, while the state-of-the-art hyperplane hashing schemes, such as $\mathrm{BH}$ [24] and $\mathrm{MH}$ [25], only conditionally deal with this problem with the assumption of data normalization. They may be arbitrarily bad without this assumption. Thus, there is no direct competitor for $\mathrm{NH}$ and $\mathrm{FH}$.

To make a fair comparison with $\mathrm{NH}$ and $\mathrm{FH}$, we implement two heuristic linear scan methods and adapt $\mathrm{BH}$ and $\mathrm{MH}$ as baselines. Specifically, we evaluate the methods described as follows.

- $\mathbf{N H}, \mathbf{F H}$, and $\mathbf{F H}^{-}$. We implement $\mathrm{NH}$ and $\mathrm{FH}$ as described in Sections 3.4 and 3.5 with the randomized sampling for the asymmetric transformation to reduce the computational cost of hash functions. To measure the effectiveness of the datadependent multi-partition strategy, we also implement the trivial $\mathrm{FH}$ (or simply $\mathrm{FH}^{-}$) as a competitor.

- NH-wo-S, FH-wo-S, and $\mathbf{F H}^{-}$-wo-S. To evaluate the effectiveness of the randomized sampling for the asymmetric transformation, we also implement $\mathrm{NH}, \mathrm{FH}$, and $\mathrm{FH}^{-}$without the randomized sampling. We call them NH-wo-S, FH-wo-S, and $\mathrm{FH}^{-}$-wo-S, respectively.

- BH and MH. To deal with the P2HNNS beyond the unit hypersphere, we adapt $\mathrm{BH}$ [24] and $\mathrm{MH}$ [25] as follows: (1) In the indexing phase, we first apply the data-dependent multipartition strategy to partition data objects into disjoint subsets; then for each subset, we normalize the data to satisfy the unit hypersphere assumption and apply $\mathrm{BH}$ and $\mathrm{MH}$ to build index accordingly. (2) In the query phase, we apply $\mathrm{BH}$ and $\mathrm{MH}$ for $k$-P2HNNS on each subset and check the number of candidates based on the fraction of data on each subset. Note that this adaptation cannot avoid the error introduced by the data normalization, but it may be more fair to compare with $\mathrm{BH}$ and $\mathrm{MH}$. For simplicity, we still call them $\mathrm{BH}$ and $\mathrm{MH}$. In addition, we consider the P2HNNS on normalized data to make a systematic comparison with $\mathrm{BH}$ and $\mathrm{MH}$.

- Random-Scan. A trivial method for $k-\mathrm{P} 2 \mathrm{HNNS}$ is to randomly select and verify data objects to determine the $k$ nearest ones. We call it Random-Scan and use it as a competitor.

- Sorted-Scan. Since P2HNNS is essentially a minimum absolute inner product search problem, a heuristic baseline beyond 

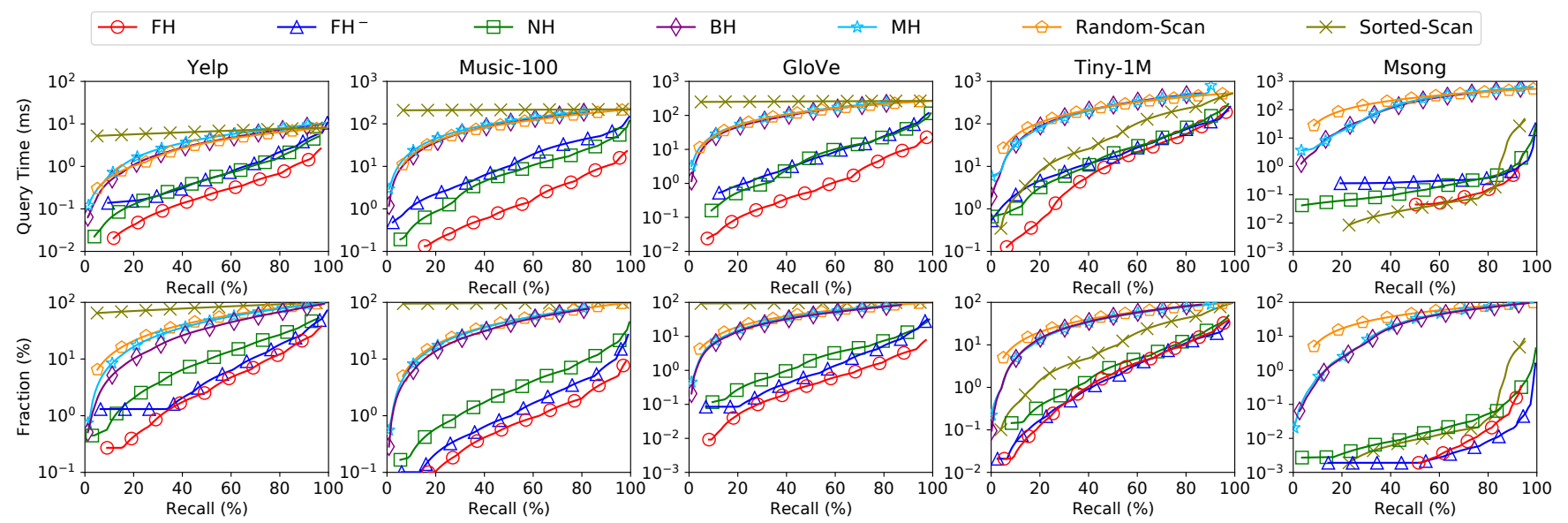

Figure 3: Query time-recall curves (top) and fraction-recall curves (bottom) of retrieving top-10 results.
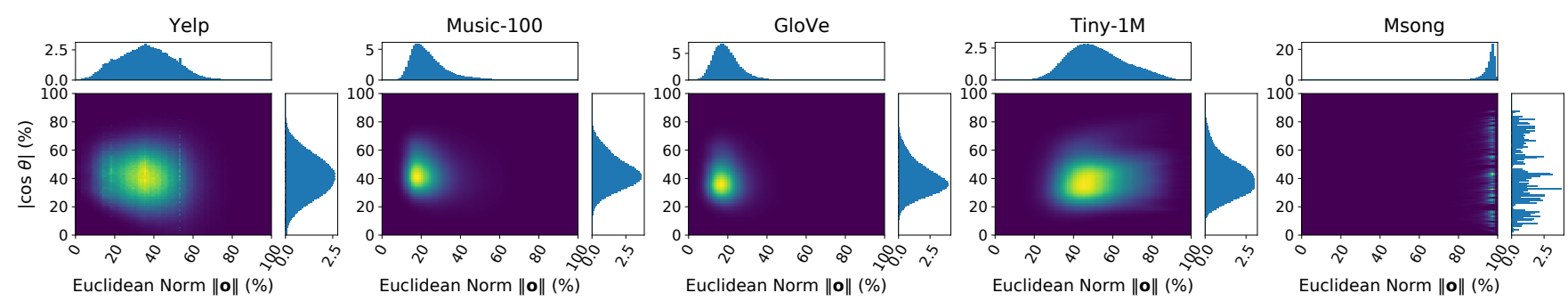

Figure 4: The heat-map of $|\cos \theta|$ and $\|o\|$ and their histograms of the five real-life datasets. The histograms of $|\cos \theta|$ and $\|o\|$ are depicted on the right and the top of the heat-map, respectively.

the Random-Scan is to sort the data objects in ascending order of their Euclidean norm and then scan them in that order. We call it Sorted-Scan and use it as a competitor either.

Since $\mathrm{BH}$ and $\mathrm{MH}$ have been proved to outperform $\mathrm{EH}$ [20], we do not choose EH as a competitor. Additionally, there are learningbased $\mathrm{BH}$ and $\mathrm{MH}$ methods that enjoy better performance than their original data-independent versions. The primary reason is that their hash functions are carefully learned and selected by some well-designed objective functions. Since the hash functions we consider in this paper are drawn i.i.d. from their hash family without learning, we do not compare with them.

For the $k$-P2HNNS, we consider $k \in\{1,5,10,20,50,100\}$. To make a fair comparison to different methods, we limit the maximum number of hash tables to 256 for all methods. Specifically, we set $t \in\{8,16,32,64,128,256\}$ for $\mathrm{NH}$ and set $m \in\{8,16,32,64,128$, $256\}$ and $l \in\{2,4,6,8,10\}$ for both $\mathrm{FH}$ and $\mathrm{FH}^{-}$. Moreover, we set $\lambda \in\{d, 2 d, 4 d, 8 d\}$ for $\mathrm{NH}, \mathrm{FH}$, and $\mathrm{FH}^{-}$. For $\mathrm{NH}$-wo-S, $\mathrm{FH}$-wo-S, and $\mathrm{FH}^{-}$-wo-S, we follow the same settings of $\mathrm{NH}, \mathrm{FH}$, and $\mathrm{FH}^{-}$ without $\lambda$. $\mathrm{BH}$ [24] and $\mathrm{MH}$ [25] apply the static $(K, L)$-bucketing strategy [8] for $k$-P2HNNS; we set $K \in\{2,4,6,8,10\}$ and $L \in$ $\{8,16,32,64,128,256\}$. Besides, $\mathrm{MH}$ needs to determine the number of random projection vectors $\left(m_{0}\right)$ for each hash function; we follow [25] and set $m_{0} \in\{4,8,16\}$. $\mathrm{NH}, \mathrm{FH}$, and $\mathrm{FH}^{-}$are not sensitive to $w$, so we simply set $w=1.0$. In addition, we observe that the effectiveness of the data-dependent multi-partition strategy mainly depends on the data distribution instead of $\sigma$. Thus, we set $\sigma=0.9$ for $\mathrm{FH}, \mathrm{BH}$, and $\mathrm{MH}$ to achieve a good performance.

\subsection{Results and Analysis}

We study the performance of $\mathrm{NH}$ and $\mathrm{FH}$ in terms of six aspects: the query performance, the indexing performance, the sensitivity to $k$, the impact of the randomized sampling, the performance on normalized data, and the impact of parameters.

Query Performance. We first study the query performance of $\mathrm{NH}$ and FH. Different number of candidates are used for different methods to achieve different recalls. To remove the impact of parameters for each method, we choose the lowest query time of a method for a certain recall from all combinations of its parameters' settings, and plot the query time-recall curves of each method in Figure 3.

$\mathrm{NH}$ and $\mathrm{FH}$ are around $3 \sim 100 \times$ faster than the best competitor in four out of five datasets (except Msong), especially when the recall falls in the range of $[20 \%, 80 \%]$. The primary reason is that $\mathrm{BH}$ and $\mathrm{MH}$ only conditionally deal with this problem. Even though we adopt the data-dependent multi-partition strategy to reduce the error caused by the data normalization, the performance of $\mathrm{BH}$ and $\mathrm{MH}$ are not arbitrarily bad but still degrade to Random-Scan. Another reason is that their $p_{1}$ and $p_{2}$ are less than $1 / 2[24,25]$. For the case $d_{P 2 H}(\boldsymbol{p}, \boldsymbol{q}) \rightarrow 0$, they need to check a large number of candidates for P2HNNS, which leads to long query time.

The patterns of all methods over Msong and Tiny-1M are different from those over the other three datasets. Referring to Equation $7, d_{P 2 H}(\boldsymbol{p}, \boldsymbol{q})=|\langle\boldsymbol{o}, \boldsymbol{q}\rangle|=\|\boldsymbol{o}\|\|\boldsymbol{q}\||\cos \theta|$, where $\theta$ is the angle between $\boldsymbol{o}$ and $\boldsymbol{q}$. Since the query results are impacted by $|\cos \theta|$ and the Euclidean norm $\|\boldsymbol{o}\|$, we plot the heat-map of $|\cos \theta|$ and $\|\boldsymbol{o}\|$ and their histograms of the five datasets in Figure 4 for illustration. 

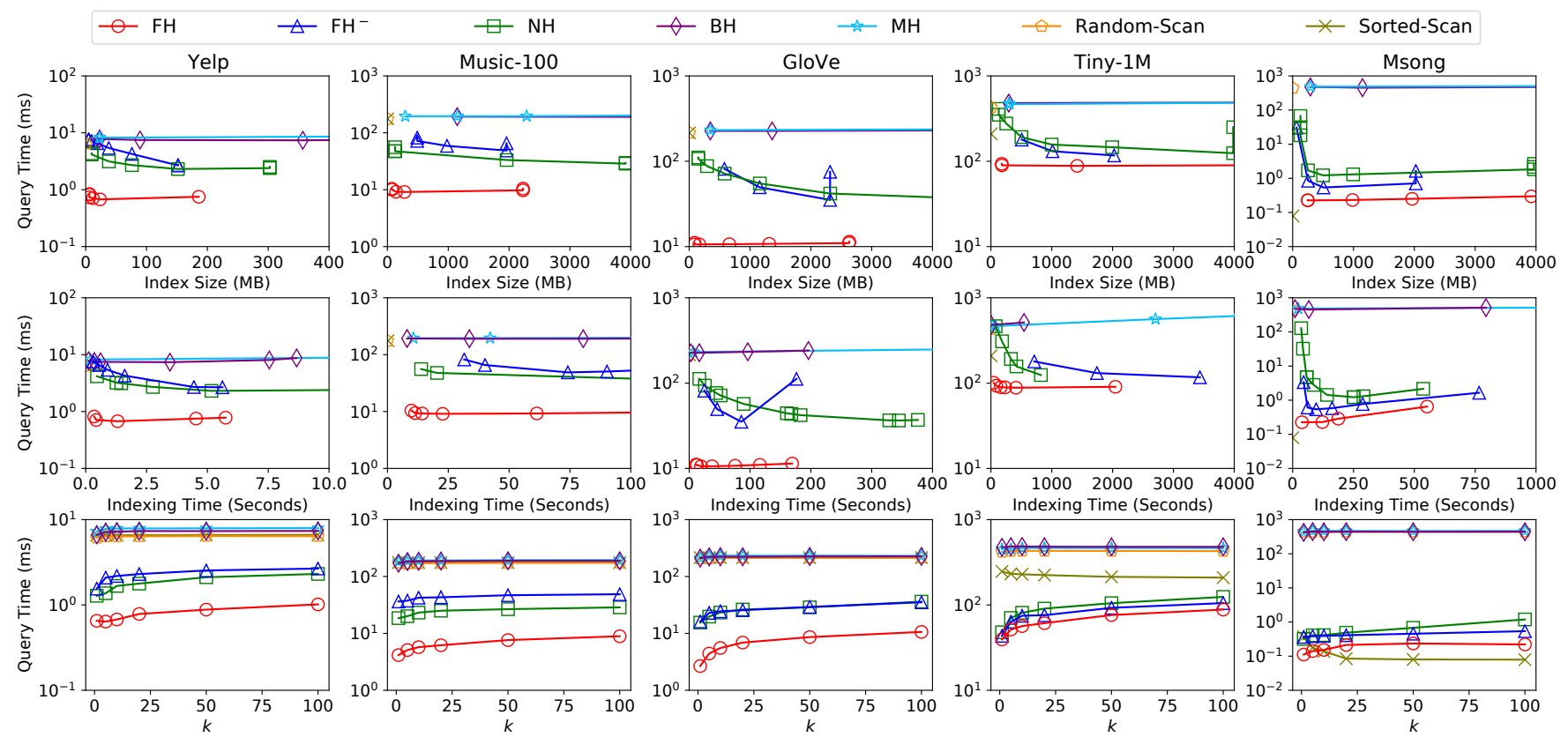

Figure 5: Query time-index size curves of retrieving top-10 results (top), query time-indexing time curves of retrieving top-10 results (middle), and query time- $k$ curves (bottom) at about $80 \%$ recall.

From Figure 3, Sorted-Scan over Msong is faster than $\mathrm{NH}$ and $\mathrm{FH}$. Moreover, Sorted-Scan over Tiny-1M is also more efficient than $\mathrm{BH}$, $\mathrm{MH}$, and Random-Scan. However, the performance of Sorted-Scan is not stable. For Yelp, Music-100, and GloVe, its performance is the worst among all methods. The reason might be the histogram of $|\cos \theta|$ of Msong (and Tiny-1M) is more average than that of Yelp, Music-100, and GloVe. Thus, Sorted-Scan that only considers the Euclidean norm but neglects the angle can perform better. Moreover, the query time of $\mathrm{BH}$ and $\mathrm{MH}$ over Msong is less than that of Random-Scan, because the Euclidean norm of Msong is more skewed than that of other datasets, which is close to the normalized case. In addition, the advantage of $\mathrm{FH}$ and $\mathrm{NH}$ over $\mathrm{BH}$ and $\mathrm{MH}$ is much more apparent. These benefits could be due to our asymmetric transformation which is more effective in distinguishing close data objects from the far-apart ones.

Compared with $\mathrm{FH}^{-}$, $\mathrm{FH}$ achieves about $10 \times$ acceleration, which verifies the effectiveness of the data-dependent multi-partition strategy in reducing false positives. Moreover, $\mathrm{FH}$ is much faster than $\mathrm{NH}$. This is because the values of $p_{1}$ and $p_{2}$ of NH differ little due to a large constant $2 M^{2}$, leading to a large $t$ and long query time. Besides, the data-dependent multi-partition strategy can speed up FH. Thus, even though NH enjoy a sub-linear query time bound, FH has a better practical performance.

To further evaluate the query performance without the impact of implementation, we also plot their fraction-recall curves in Figure 3. The fraction-recall curves show similar trends to the query timerecall curves, which indicates $\mathrm{FH}$ and $\mathrm{NH}$ (especially $\mathrm{FH}$ ) can check much less candidates than $\mathrm{BH}$ and $\mathrm{MH}$ for the same recalls.

Indexing Performance. We then study the indexing performance of $\mathrm{NH}$ and $\mathrm{FH}$. We plot the query time-index size (and indexing time) curves of all methods at about $80 \%$ recall in Figure 5. Each point in those curves refers to the query time and index size (or indexing time) of a method with a certain combination of their parameters whose recall is about $80 \%$. Similar trends can be observed from other recalls.

From Figure 5, NH and FH enjoy better trade-off between query time and space than $\mathrm{BH}$ and $\mathrm{MH}$. As we discussed before, $\mathrm{BH}$ and $\mathrm{MH}$ suffer from the low collision probability, so they require much more space and query time than $\mathrm{NH}$ and $\mathrm{FH}$ to achieve a certain recall. Compared with $\mathrm{BH}$ and $\mathrm{MH}$, the asymmetric transformation we proposed will increase the data dimension from $d$ to $O\left(d^{2}\right)$, but we use the randomized sampling to reduce the computational cost of hash functions. Thus, the indexing time of $\mathrm{NH}$ and $\mathrm{FH}$ can be lower than that of $\mathrm{BH}$ and $\mathrm{MH}$. Moreover, $\mathrm{MH}$ requires longer indexing time than $\mathrm{BH}$, because it requires computing $m_{0}\left(m_{0} \geq 2\right)$ random projection vectors to determine a hash value. In addition, for some methods such as FH, increasing hash tables cannot always reduce the query time. It is probably because certain indexing overhead is good enough for $\mathrm{FH}$ to achieve $80 \%$ recall.

Sensitivity to $k$. We consider $k \in\{1,5,10,20,50,100\}$ and study the sensitivity to $k$ for each method. We plot the query time- $k$ curves of all methods at about $80 \%$ recall in Figure 5. Similar trends can be observed from other recalls.

From Figure 5, NH and $\mathrm{FH}$ is much faster than other competitors. However, the slope of $\mathrm{NH}$ and $\mathrm{FH}$ is slightly larger than that of $\mathrm{BH}$ and $\mathrm{MH}$ when $k$ is small, e.g., $k<20$, because $\mathrm{BH}$ and $\mathrm{MH}$ are inefficient and hence they are not sensitive to $k$. As $k$ increases, e.g., $k \geq 50$, the slope of $\mathrm{NH}$ and $\mathrm{FH}$ becomes as small as that of other competitors. This observation indicates that the performance of $\mathrm{NH}$ and $\mathrm{FH}$ becomes more and more stable when $k$ increases.

Impact of Randomized Sampling. Next, we study the impact of the randomized sampling for the asymmetric transformations of 

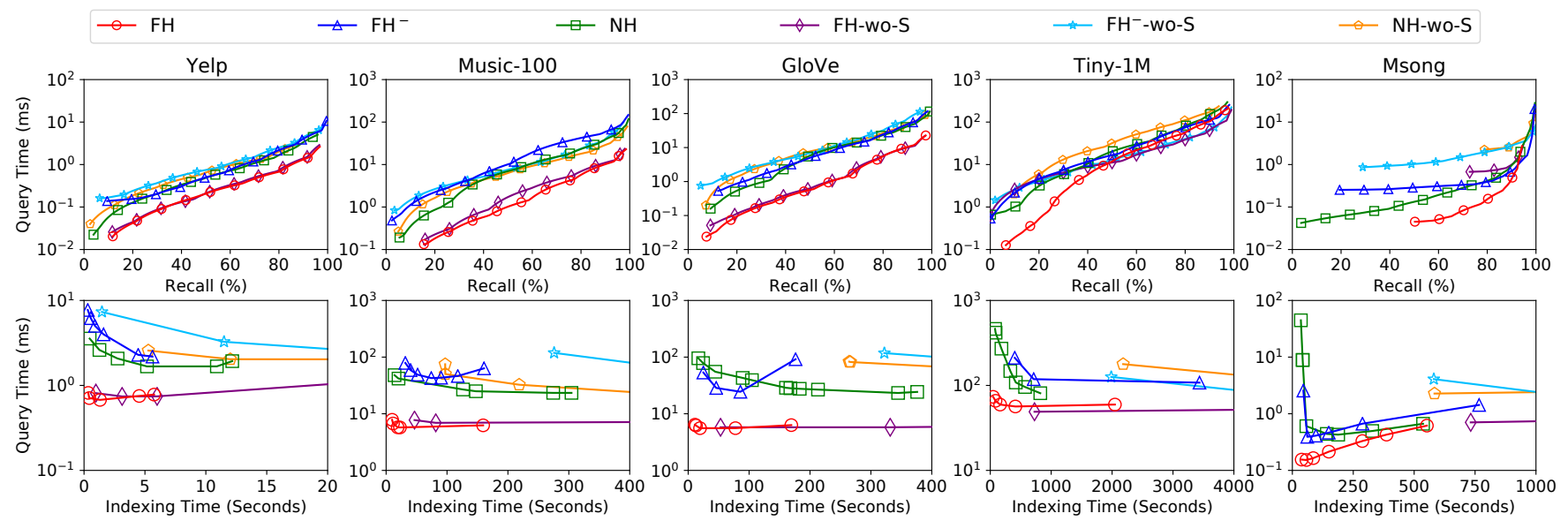

Figure 6: Query time-recall curves of retrieving top-10 results (top) and query time-indexing time curves of retrieving top-10 results at about $80 \%$ recall (bottom).

$\mathrm{NH}$ and FH. Figure 6 shows the query time-recall curves and the query time-indexing time curves at about $80 \%$ recall of $\mathrm{NH}, \mathrm{FH}$, $\mathrm{FH}^{-}$, and their variants without randomized sampling.

The query performance of $\mathrm{FH}, \mathrm{FH}^{-}$, and $\mathrm{NH}$ is comparable to that of $\mathrm{FH}$-wo-S, $\mathrm{FH}^{-}$-wo-S, and $\mathrm{NH}$-wo-S, which demonstrates the effectiveness of the randomized sampling, in the sense that the data objects after the asymmetric transformation can be effectively reconstructed by the randomized sampling. Moreover, since the randomized sampling reduces the data dimension from $O\left(d^{2}\right)$ to $O\left(1 / \epsilon^{2}\right)$, the query time of $\mathrm{NH}, \mathrm{FH}$, and $\mathrm{FH}^{-}$may be lower than that of $\mathrm{FH}$-wo-S, $\mathrm{FH}^{-}$-wo-S, and $\mathrm{NH}$-wo-S. For example, the query time of $\mathrm{FH}$ over Msong is much lower than that of FH-wo-S.

Due to the use of pipeline computation, we can avoid to store the coordinates of $\boldsymbol{P} \circ f\left(\boldsymbol{o}_{\boldsymbol{i}}\right)$. Thus, the index sizes of $\mathrm{FH}, \mathrm{FH}^{-}$, and $\mathrm{NH}$ are identical to those of $\mathrm{FH}$-wo-S, $\mathrm{FH}^{-}$-wo-S, and $\mathrm{NH}^{-}$ wo-S. However, their indexing time is quite different. Since the randomized sampling reduces the data dimension from $O\left(d^{2}\right)$ to $O\left(1 / \epsilon^{2}\right), \mathrm{FH}, \mathrm{FH}^{-}$, and $\mathrm{NH}$ enjoy much lower indexing time than FH-wo-S, $\mathrm{FH}^{-}$-wo-S, and $\mathrm{NH}$-wo-S, respectively.

Performance on Normalized Data. To make a fair comparison with $\mathrm{BH}$ and $\mathrm{MH}$, we further normalize the datasets to the unit hypersphere and study the performance of $\mathrm{NH}$ and FH. Figure 7 shows the query time-recall curves and query time-indexing time curves at about $80 \%$ recall of $\mathrm{NH}, \mathrm{FH}, \mathrm{BH}$, and $\mathrm{MH}$.

Compared to Figure 3, the query performance of $\mathrm{NH}$ and $\mathrm{FH}$ degrade, especially for $\mathrm{NH}$. This might be because after data normalization, it is hard to distinguish the close data objects from the far-apart ones. Moreover, the query performance of $\mathrm{BH}$ and $\mathrm{MH}$ do not improve very much. The primary reason is that when $d_{P 2 H}(\boldsymbol{p}, \boldsymbol{q}) \rightarrow 0, \rho=\lim _{d_{P 2 H} \rightarrow 0} \ln \left(1 / p_{1}\right) / \ln \left(1 / p_{2}\right)=1$, leading to $L=O(n)$ hash tables. Due to their limited improvements, $\mathrm{NH}$ and $\mathrm{FH}$ are still better than $\mathrm{BH}$ and $\mathrm{MH}$. The patterns of the four methods over Msong and Tiny-1M are different from those over the other three datasets, because after data normalization, the results of P2HNNS are only impacted by $|\cos \theta|$ and the histograms of $|\cos \theta|$ of Msong and Tiny-1M are different from those of other three datasets. The query time-indexing time curves of $\mathrm{NH}, \mathrm{FH}, \mathrm{BH}$, and $\mathrm{MH}$ in Figure 7 are similar to that in Figure 5. The reason is that the data normalization does not impact the indexing time.

Impacts of Parameters. Finally, we study the impacts of the parameters of $\mathrm{NH}$ and $\mathrm{FH}$. We only show results over GloVe. Similar trends can be observed from other datasets.

Impacts of $t$ and $\lambda$ for $\mathrm{NH}$. We first study the impact of the number of hash tables $t$ for NH. We fix $\lambda=2 d$. Figure 8 shows the query time-recall curves of $\mathrm{NH}$ for $t \in\{8,16,32,64,128,256\}$. NH achieves the lowest query time under different recalls with different $t$ 's. Thus, the optimal $t$ value for $\mathrm{NH}$ under different recalls is different. In general, a larger $t$ leads to lower query time of $\mathrm{NH}$ especially when the recalls are high.

We then study the impact of $\lambda$ for NH. We fix $t=256$. Figure 8 shows the query time-recall curves of $\mathrm{NH}$ for $\lambda \in\{1 d, 2 d, 4 d, 8 d\}$. From Figure 8 , the query performance of $\mathrm{NH}$ under different settings of $\lambda$ is close to each other. Thus, the query performance is not sensitive to $\lambda$. The reason may be that with a larger value of $\lambda$, the data objects may be reconstructed better, but it also introduces longer query time to compute hash values. Since a larger value of $\lambda$ will lead to larger indexing time, setting a small $\lambda$ value (e.g., $1 d$ or $2 d$ ) is suitable to $\mathrm{NH}$.

Impacts of $m, l$, and $\lambda$ for $F H$. We then study the impacts of $m, l$, and $\overline{\lambda \text { for FH. We first fix } \lambda=2 d}$ and $l=4$. Figure 8 shows the query time-recall curves of FH for $m=\{8,16,32,64,128,256\}$. The query performance of $\mathrm{FH}$ is not very sensitive to $m$. For the low recalls, i.e., the recall value is less than $25 \%$, FH with small $m$ 's is slightly better than that with large $m$ 's. Since a larger $m$ will lead to a larger indexing overhead, setting $m=16$ or $m=32$ is suitable to FH.

Next, we fix $\lambda=2 d$ and $m=16$. Figure 8 shows the query timerecall curves of $\mathrm{FH}$ for $l \in\{2,4,6,8,10\}$. For the high recalls, except for $l=10$, the query time of FH under other settings of $l$ is close to each other, while for the low recalls, i.e., the recall is less than $25 \%$, the query time of FH with small l's is slightly lower than that with large $l$ 's. Thus, setting a small $l$ value (e.g., 2 or 4 ) is satisfied for $\mathrm{FH}$. In addition, the impact of $\lambda$ for $\mathrm{FH}$ is similar to that for $\mathrm{NH}$, and the query performance of $\mathrm{FH}$ is not sensitive to $\lambda$. 

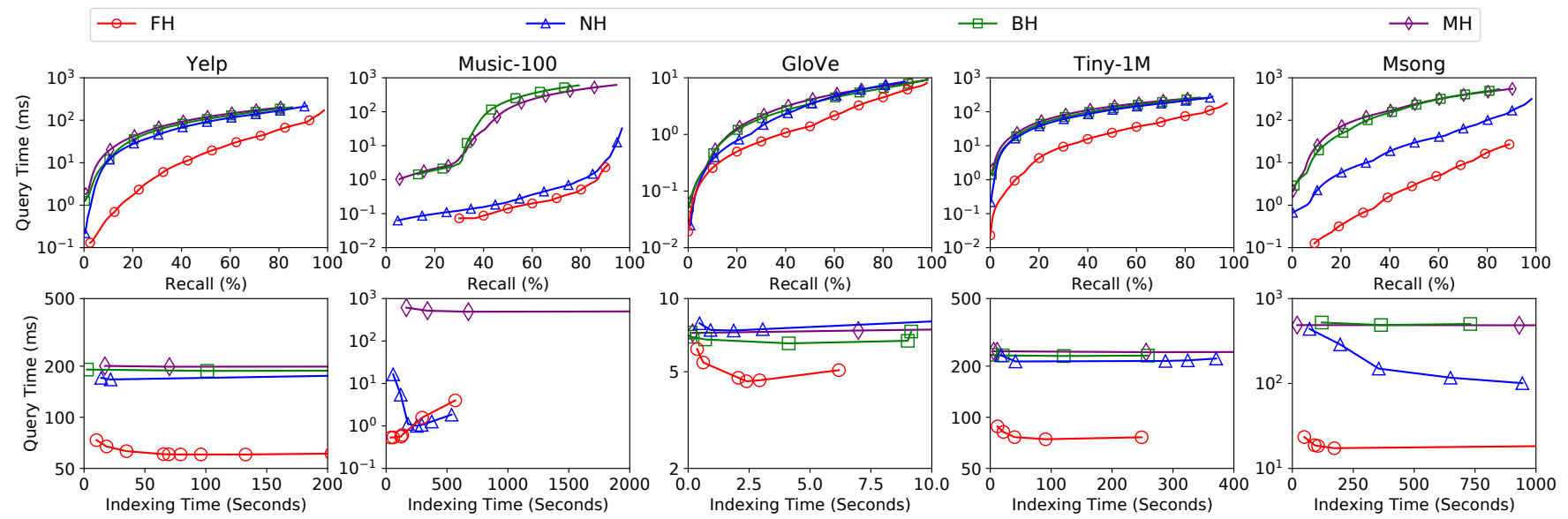

Figure 7: Results on normalized datasets: query time-recall curves of retrieving top-10 results (top) and query time-indexing time curves of retrieving top-10 results at about $80 \%$ recall (bottom).
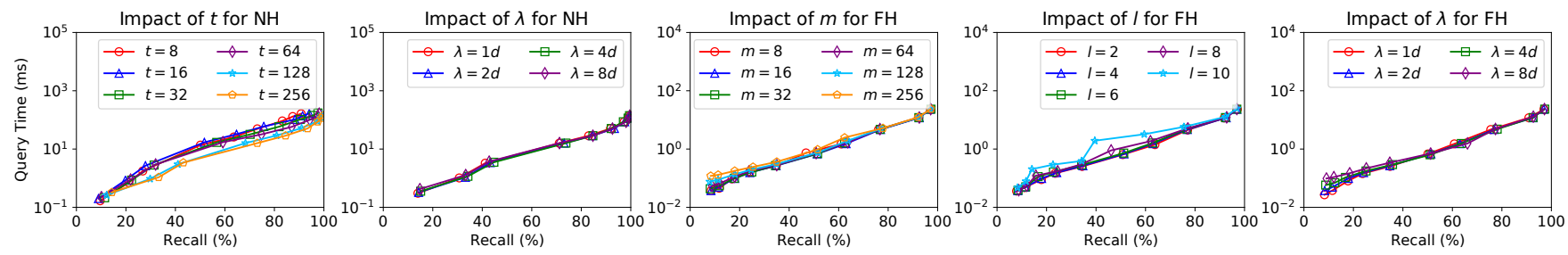

Figure 8: Impacts of the parameters of $\mathrm{NH}$ and FH over GloVe.

\section{CONCLUSION}

In this paper, we investigate a very challenging problem of P2HNNS. We design a novel asymmetric transformation and propose the first two provable schemes $\mathrm{NH}$ and FH for high-dimensional P2HNNS beyond the unit hypersphere. Like a coin has two sides, this transformation is not perfect, leading to more space and computational cost. We develop a pipeline computation to avoid $O\left(n d^{2}\right)$ space to store the intermediate values of data objects; moreover, we propose to use the randomized sampling to approximate this transformation that can reduce the computational cost of hash functions from $O\left(d^{2}\right)$ to $O\left(1 / \epsilon^{2}\right)$. With this transformation, the hash functions of $\mathrm{NH}$ and $\mathrm{FH}$ are locality-sensitive to the hyperplane queries. We further show that NH can deal with the P2HNNS in sub-linear time, while $\mathrm{FH}$ enjoys the better practical performance. Extensive experiments over five real-life datasets confirm their superior performance.

\section{ACKNOWLEDGMENTS}

This research is supported by the National Research Foundation, Singapore under its Strategic Capability Research Centres Funding Initiative. Any opinions, findings and conclusions or recommendations expressed in this material are those of the author(s) and do not reflect the views of National Research Foundation, Singapore.

\section{A HYPERPLANE QUERY GENERATION}

Suppose $\left[l_{1}, r_{1}\right], \cdots,\left[l_{d-1}, r_{d-1}\right]$ are the ranges of coordinates of data points such that $l_{i} \leq p_{i} \leq r_{i}$ for all $\boldsymbol{p}=\left(p_{1}, \cdots, p_{d-1}\right) \in$ $\mathcal{D} \subset \mathbb{R}^{d-1}$, where $i \in\{1, \cdots, d-1\}$. Without loss of generality, we assume the datasets satisfy the following two conditions: (1) the range of coordinates for all $\boldsymbol{p} \in \mathcal{D}$ is symmetric, i.e., $l_{i}=-r_{i}$ for each $i \in\{1, \cdots, d-1\}$; (2) all $\boldsymbol{p} \in \mathcal{D}$ are bounded inside the unit hypersphere, i.e., $\|\boldsymbol{p}\| \leq 1$ for all $\boldsymbol{p} \in \mathcal{D}$.

We can shift and rescale the data points if the real-life datasets do not satisfy these two conditions, i.e., $\boldsymbol{p}^{\prime}=(\boldsymbol{p}-\overline{\boldsymbol{p}}) / \Omega$ for all $\boldsymbol{p} \in \mathcal{D}$, where $\Omega=\max _{\boldsymbol{p} \in \mathcal{D}}\|\boldsymbol{p}-\overline{\boldsymbol{p}}\|$ and $\overline{\boldsymbol{p}}=\left(\frac{l_{1}+r_{1}}{2}, \cdots, \frac{l_{d-1}+r_{d-1}}{2}\right)$ is the center of all data points. Notably, we do not assume all $\boldsymbol{p} \in \mathcal{D}$ are normalized or change the distortion between the data points, i.e., $\frac{\|\boldsymbol{p}-\boldsymbol{u}\|}{\|\boldsymbol{p}-\boldsymbol{v}\|}=\frac{\left\|\boldsymbol{p}^{\prime}-\boldsymbol{u}^{\prime}\right\|}{\left\|\boldsymbol{p}^{\prime}-\boldsymbol{v}^{\prime}\right\|}$ for any three $\boldsymbol{p}, \boldsymbol{u}, \boldsymbol{v} \in \mathcal{D}$. Then, we generate a random hyperplane query $\boldsymbol{q} \in \mathbb{R}^{d}$ as follows:

(1) We generate a random vector $\boldsymbol{w}_{\boldsymbol{q}}=\left(q_{1}, \cdots, q_{d-1}\right)$ as a normal vector to the query hyperplane, i.e., we generate $q_{i}$ uniformly at random from $\left[l_{i}, r_{i}\right]$ for each $i \in\{1, \cdots, d-1\}$;

(2) We generate a random point $x=\left(x_{1}, \cdots, x_{d-1}\right)$ as a pivot which is confined to be in the query hyperplane, i.e., we generate each $x_{i}$ i.i.d. from $\left[l_{i}, r_{i}\right]$, where $i \in\{1, \cdots, d-1\}$;

(3) We construct $\boldsymbol{q}=\left(\boldsymbol{w}_{\boldsymbol{q}} ; q_{d}\right)=\left(q_{1}, \cdots, q_{d-1}, q_{d}\right)$ based on $\boldsymbol{w}_{\boldsymbol{q}}$ and $x$, where $q_{d}=-\sum_{i=1}^{d-1} x_{i} q_{i}$.

Since each $x_{i}$ satisfies the inequality $l_{i} \leq x_{i} \leq r_{i}$, the hyperplane query $\boldsymbol{q}$ is very likely to divide the dataset into two parts, which helps us simulate the scenarios for the real-life applications, e.g., large-scale active learning with SVMs. Moreover, since we assume the datasets are bounded inside the unit hypersphere, $q_{d}$ is restricted in a small range. Thus, when we apply $P \circ f(\cdot)$ and $Q \circ g(\cdot)$ (or $\left.Q^{\prime} \circ g(\cdot)\right)$ to convert the P2HNNS into the classic NNS (or FNS), the distortion error between data points can be largely reduced, i.e., $\left|\frac{\|p-u\|}{\|p-v\|}-\frac{\|P \circ f(p)-P \circ f(u)\|}{\|P \circ f(p)-P \circ f(v)\|}\right| \rightarrow 0$ for any three $\boldsymbol{p}, \boldsymbol{u}, \boldsymbol{v} \in \mathcal{D}$. 


\section{REFERENCES}

[1] Alexandr Andoni and Piotr Indyk. 2006. Near-optimal hashing algorithms for approximate nearest neighbor in high dimensions. In FOCS. 459-468.

[2] Alexandr Andoni, Piotr Indyk, Thijs Laarhoven, Ilya Razenshteyn, and Ludwig Schmidt. 2015. Practical and optimal LSH for angular distance. In NeurIPS. 12251233.

[3] Alexandr Andoni and Ilya Razenshteyn. 2015. Optimal data-dependent hashing for approximate near neighbors. In STOC. 793-801.

[4] Martin Aumüller, Tobias Christiani, Rasmus Pagh, and Francesco Silvestri. 2018 Distance-sensitive hashing. In PODS. 89-104.

[5] Yoram Bachrach, Yehuda Finkelstein, Ran Gilad-Bachrach, Liran Katzir, Noam Koenigstein, Nir Nice, and Ulrich Paquet. 2014. Speeding up the xbox recommender system using a euclidean transformation for inner-product spaces. In RecSys. 257-264.

[6] Jon Louis Bentley. 1990. K-d trees for semidynamic point sets. In SoCG. 187-197.

[7] Kevin Beyer, Jonathan Goldstein, Raghu Ramakrishnan, and Uri Shaft. 1999. When is "nearest neighbor" meaningful?. In ICDT. 217-235.

[8] Moses S Charikar. 2002. Similarity estimation techniques from rounding algorithms. In STOC. 380-388.

[9] Ryan R Curtin, Javier Echauz, and Andrew B Gardner. 2019. Exploiting the structure of furthest neighbor search for fast approximate results. Information Systems 80 (2019), 124-135.

[10] Ryan R Curtin and Andrew B Gardner. 2016. Fast approximate furthest neighbors with data-dependent candidate selection. In International Conference on Similarity Search and Applications. 221-235.

[11] Mayur Datar, Nicole Immorlica, Piotr Indyk, and Vahab S Mirrokni. 2004. Locality sensitive hashing scheme based on p-stable distributions. In SoCG. 253-262.

[12] Junhao Gan, Jianlin Feng, Qiong Fang, and Wilfred Ng. 2012. Locality-sensitive hashing scheme based on dynamic collision counting. In SIGMOD. 541-552.

[13] Sariel Har-Peled, Piotr Indyk, and Rajeev Motwani. 2012. Approximate nearest neighbor: Towards removing the curse of dimensionality. Theory of Computing 8,1 (2012), 321-350.

[14] Qiang Huang, Jianlin Feng, Qiong Fang, and Wilfred Ng. 2017. Two Efficient Hashing Schemes for High-Dimensional Furthest Neighbor Search. TKDE 29, 12 (2017), 2772-2785

[15] Qiang Huang, Jianlin Feng, Qiong Fang, Wilfred Ng, and Wei Wang. 2017. Query aware locality-sensitive hashing scheme for $l_{p}$ norm. The VLDB fournal 26, 5 (2017), 683-708.

[16] Qiang Huang, Jianlin Feng, Yikai Zhang, Qiong Fang, and Wilfred Ng. 2015 Query-aware locality-sensitive hashing for approximate nearest neighbor search. PVLDB 9, 1 (2015), 1-12.

[17] Oiang Huang, Guihong Ma, Jianlin Feng, Oiong Fang, and Anthony KH Tung. 2018. Accurate and Fast Asymmetric Locality-Sensitive Hashing Scheme for Maximum Inner Product Search. In KDD. 1561-1570.

[18] Piotr Indyk. 2003. Better algorithms for high-dimensional proximity problems via asymmetric embeddings. In SODA. 539-545.

[19] Piotr Indyk and Rajeev Motwani. 1998. Approximate nearest neighbors: towards removing the curse of dimensionality. In STOC. 604-613.

[20] Prateek Jain, Sudheendra Vijayanarasimhan, and Kristen Grauman. 2010. Hashing hyperplane queries to near points with applications to large-scale active learning. In NeurIPS. 928-936.

[21] Yifan Lei, Qiang Huang, Mohan Kankanhalli, and Anthony Tung. 2019. Sublinear Time Nearest Neighbor Search over Generalized Weighted Space. In ICML. 37733781.

[22] Yifan Lei, Qiang Huang, Mohan Kankanhalli, and Anthony KH Tung. 2020 Locality-Sensitive Hashing Scheme based on Longest Circular Co-Substring. In SIGMOD. 2589-2599.

[23] Wanqi Liu, Hanchen Wang, Ying Zhang, Wei Wang, and Lu Qin. 2019. I-LSH: I/O Efficient c-Approximate Nearest Neighbor Search in High-Dimensional Space. In IEEE ICDE. 1670-1673.

[24] Wei Liu, Jun Wang, Yadong Mu, Sanjiv Kumar, and Shih-Fu Chang. 2012. Compact hyperplane hashing with bilinear functions. In ICML. 467-474.
[25] Xianglong Liu, Xinjie Fan, Cheng Deng, Zhujin Li, Hao Su, and Dacheng Tao. 2016. Multilinear hyperplane hashing. In CVPR. 5119-5127.

[26] Kejing Lu and Mineichi Kudo. 2020. R2LSH: A Nearest Neighbor Search Scheme Based on Two-dimensional Projected Spaces. In IEEE ICDE. 1045-1056.

[27] Kejing Lu, Hongya Wang, Wei Wang, and Mineichi Kudo. 2020. VHP: Approximate Nearest Neighbor Search via Virtual Hypersphere Partitioning. PVLDB 13, 9 (2020), 1443-1455.

[28] Behnam Neyshabur and Nathan Srebro. 2015. On Symmetric and Asymmetric LSHs for Inner Product Search. In ICML. 1926-1934

[29] Stephen M Omohundro. 1989. Five balltree construction algorithms. International Computer Science Institute Berkeley.

[30] Rasmus Pagh, Francesco Silvestri, Johan Sivertsen, and Matthew Skala. 2015. Approximate furthest neighbor in high dimensions. In International Conference on Similarity Search and Applications. 3-14.

[31] Rasmus Pagh, Francesco Silvestri, Johan Sivertsen, and Matthew Skala. 2017. Approximate furthest neighbor with application to annulus query. Information Systems 64 (2017), 152-162.

[32] Parikshit Ram and Alexander G Gray. 2012. Maximum inner-product search using cone trees. In KDD. 931-939.

[33] Mohammad Saberian, Jose Costa Pereira, Can Xu, Jian Yang, and Nuno Nvasconcelos. 2016. Large margin discriminant dimensionality reduction in prediction space. In NeurIPS. 1488-1496.

[34] Greg Schohn and David Cohn. 2000. Less is More: Active Learning with Support Vector Machines. In ICML. 839-846.

[35] Anshumali Shrivastava and Ping Li. 2014. Asymmetric LSH (ALSH) for sublinear time Maximum Inner Product Search (MIPS). In NeurIPS. 2321-2329.

[36] Anshumali Shrivastava and Ping Li. 2015. Improved asymmetric locality sensitive hashing (ALSH) for Maximum Inner Product Search (MIPS). In UAI. 812-821

[37] Yifang Sun, Wei Wang, Jianbin Qin, Ying Zhang, and Xuemin Lin. 2014. SRS: solving c-approximate nearest neighbor queries in high dimensional euclidean space with a tiny index. PVLDB 8, 1 (2014), 1-12.

[38] Yufei Tao, Ke Yi, Cheng Sheng, and Panos Kalnis. 2009. Quality and efficiency in high dimensional nearest neighbor search. In SIGMOD. 563-576.

[39] Simon Tong and Daphne Koller. 2001. Support vector machine active learning with applications to text classification. $7 M L R 2$, Nov (2001), 45-66.

[40] Antonio Torralba, Rob Fergus, and William T Freeman. 2008. 80 million tiny images: A large data set for nonparametric object and scene recognition. TPAMI 30, 11 (2008), 1958-1970.

[41] Sudheendra Vijayanarasimhan and Kristen Grauman. 2014. Large-scale live active learning: Training object detectors with crawled data and crowds. IfCV 108, 1-2 (2014), 97-114

[42] Sudheendra Vijayanarasimhan, Prateek Jain, and Kristen Grauman. 2014. Hashing hyperplane queries to near points with applications to large-scale active learning. TPAMI 36, 2 (2014), 276-288.

[43] Runhui Wang and Dong Deng. 2020. DeltaPQ: lossless product quantization code compression for high dimensional similarity search. PVLDB 13, 13 (2020), 3603-3616.

[44] Roger Weber, Hans-Jörg Schek, and Stephen Blott. 1998. A quantitative analysis and performance study for similarity-search methods in high-dimensional spaces. In $V L D B$, Vol. 98. 194-205.

[45] Chang Xu, Dacheng Tao, Chao Xu, and Yong Rui. 2014. Large-margin weakly supervised dimensionality reduction. In ICML. 865-873.

[46] Xiao Yan, Jinfeng Li, Xinyan Dai, Hongzhi Chen, and James Cheng. 2018. NormRanging LSH for Maximum Inner Product Search. In NeurIPS. 2956-2965.

[47] Teng Zhang and Zhi-Hua Zhou. 2018. Optimal margin distribution clustering. In AAAI. 4474-4481.

[48] Bin Zhao, Fei Wang, and Changshui Zhang. 2008. Efficient maximum margin clustering via cutting plane algorithm. In SDM. 751-762.

[49] Bolong Zheng, Xi Zhao, Lianggui Weng, Nguyen Quoc Viet Hung, Hang Liu, and Christian S Jensen. 2020. PM-LSH: A fast and accurate LSH framework for high-dimensional approximate NN search. PVLDB 13, 5 (2020), 643-655.

[50] Yuxin Zheng, Qi Guo, Anthony KH Tung, and Sai Wu. 2016. Lazylsh: Approximate nearest neighbor search for multiple distance functions with a single index. In SIGMOD. 2023-2037. 